Homology, Homotopy and Applications, vol.10(3), 2008, pp.193-221

\title{
DIAGRAMS INDEXED BY GROTHENDIECK CONSTRUCTIONS
}

\author{
SHARON HOLLANDER
}

\author{
(communicated by Haynes R. Miller)
}

\begin{abstract}
Let $I$ be a small indexing category, $G: I^{o p} \rightarrow$ Cat be a functor and $B G \in \mathcal{C}$ at denote the Grothendieck construction on $G$. We define and study Quillen pairs between the category of diagrams of simplicial sets (resp. categories) indexed on $B G$ and the category of $I$-diagrams over $N(G)$ (resp. $G$ ). As an application we obtain a Quillen equivalence between the categories of presheaves of simplicial sets (resp. groupoids) on a stack $\mathcal{M}$ and presheaves of simplicial sets (resp. groupoids) over $\mathcal{M}$.
\end{abstract}

\section{Introduction}

The motivation for this paper was the study of homotopy theory of (pre)sheaves on a stack. Since the site associated to a stack $\mathcal{M}$ is a Grothedieck construction this led us to an investigation of the homotopy theory of diagrams indexed on a category which is itself a Grothendieck construction (of a diagram of small categories). The body of the paper is concerned with analyzing various Quillen pairs between diagram categories. These adjunctions are of general interest and we present some examples not related to the theory of stacks. We conclude the paper with the applications to stacks.

Stacks were introduced in algebraic geometry in order to parametrize families of objects when the presence of automorphisms prevented representability by a scheme or even a sheaf $[\mathbf{A}, \mathbf{D M}, \mathbf{G i}]$. Recently stacks have come to play an important role in algebraic topology. Complex oriented cohomology theories give rise to stacks over the moduli stack of formal groups and in certain situations, conversely, stacks over the moduli stack of formal groups give rise to spectra $[\mathbf{G}, \mathbf{R 2}, \mathbf{G H M R}, \mathbf{B}]$. One fundamental example is the spectrum of topological modular forms $[\mathbf{H p}]$ which is associated to the moduli stack of elliptic curves.

Classically, stacks were defined as categories fibered in groupoids over a site $\mathcal{C}$ which satisfy descent $[\mathbf{D M}$, Definition 4.1]. In $[\mathbf{H}]$ we show that a category fibered in groupoids $F$ over $\mathcal{C}$ is a stack if and only if the assignment satisfies the homotopy sheaf

The author is supported by a postdoctoral fellowship from CAMGSD and the POCI-Research Units Pluriannual Funding Program.

Received June 7, 2007, revised November 13, 2007; published on December 5, 2008.

2000 Mathematics Subject Classification: 18G55, 55P99.

Key words and phrases: Grothendieck construction, stacks, homotopy theory of diagrams.

This article is available at http://intlpress.com/HHA/v10/n3/a10

Copyright (C) 2008, International Press. Permission to copy for private use granted. 
condition; that is, for each cover $\left\{U_{i} \rightarrow X \in \mathcal{C}\right\}$, the natural map

$$
F(X) \stackrel{\sim}{\longrightarrow} \operatorname{holim}\left(\prod F\left(U_{i}\right) \Longrightarrow \prod F\left(U_{i j}\right) \Longrightarrow \prod F\left(U_{i j k}\right) \cdots\right)
$$

is an equivalence of categories. This characterization of stacks naturally leads to a model structure on categories fibered in groupoids over $\mathcal{C}$, in which the fibrant objects are the stacks. Similarly, one can consider the strict functors, or presheaves of groupoids on $\mathrm{C}$, denoted $P(\mathcal{C}, \mathcal{G r p d})$. Here too there is a local model structure, denoted $P(\mathcal{C}, \mathcal{G} r p d)_{L}$, in which the fibrant objects are those functors which are stacks or, equivalently, satisfy the homotopy sheaf condition. Furthermore, there is a Quillen equivalence between these two model categories (see [H, Section 4]).

For the purposes of this paper it makes no difference which of the Quillen equivalent model categories one chooses to work in, and for the sake of simplicity we will work in $P(\mathcal{C}, \mathcal{G r p d})_{L}$.

Given a stack $\mathcal{M}$ on $\mathcal{C}$, a sheaf on $\mathcal{M}$ [DM, Definition 4.10] is a sheaf on a site $\mathcal{C} / \mathcal{M}$ whose objects are morphisms $X \rightarrow \mathcal{M} \in P(\mathcal{C}, \mathcal{G r p d})$ with $X \in \mathcal{C}$, and morphisms are triangles with a commuting homotopy. ${ }^{1}$ Covers in $\mathcal{C} / \mathcal{M}$ are the collections of morphisms which forget to covers in $\mathcal{C}$. The site $\mathcal{C} / \mathcal{M}$ makes sense for any presheaf of groupoids $\mathcal{M}$ (or even a presheaf of categories) on a site $\mathcal{C}$ (see [H2, Section 2.1]). If $\mathcal{M}$ is represented by an object $X \in \mathcal{C}$, then the site described above is just the usual topology on the over category $\mathrm{e} / X$.

The Grothendieck construction on a functor $F: I^{o p} \rightarrow$ Cat is the category with objects pairs $(i, a)$ with $i \in I$ and $a \in F(i)$ and morphisms $(i, a) \rightarrow(j, b)$ pairs $(\alpha, \phi)$ with $\alpha: i \rightarrow j$ and $\phi: a \rightarrow \alpha^{*}(b)$. So the underlying category of $\mathrm{C} / \mathcal{M}$ is, by definition, just the Grothendieck construction on the functor $\mathcal{M}: \mathcal{C}^{o p} \rightarrow \operatorname{Grpd}$.

Since we are interested in understanding the homotopy theory of (pre)sheaves on a stack $\mathcal{M}$ and $\mathcal{C} / \mathcal{M}$ is a Grothendieck construction, this leads naturally to considering the homotopy theory of diagrams indexed by Grothendieck constructions. The Grothendieck construction on $F: I^{o p} \rightarrow$ Cat is the coend in Cat:

$$
B F=(I /-) \otimes_{I} F \stackrel{\text { def }}{=} \operatorname{coeq}\left(\coprod_{i \rightarrow j \in I}(I / i) \times F(j) \Rightarrow \coprod_{i \in I}(I / i) \times F(i)\right) .
$$

Our first observations are that there are adjunctions (see Propositions 2.1 and 3.2)

$$
\begin{aligned}
& B: \mathcal{C} a t^{o p} \leftrightarrow \mathcal{C} a t / I: \Gamma, \\
& p: \mathcal{C} a t / I \leftrightarrow \mathrm{Cat}^{I^{o p}}: B .
\end{aligned}
$$

Cat has a cofibrantly generated model structure where weak equivalences are equivalences of categories $[\mathbf{R}]$. This determines model structures on the above categories for which the adjunctions above are Quillen pairs, and Quillen equivalences if $I$ is a groupoid (see Theorem 2.7 and Proposition 3.7). An application of the first adjunction is a characterization of fibered categories over a category $\mathcal{C}$ as those categories over $\mathcal{C}$ which are equivalent to $B G \rightarrow \mathcal{C}$ for some functor $G$ : $\mathcal{C}^{o p} \rightarrow \mathcal{C}$ at (Proposition 2.16).

\footnotetext{
${ }^{1}$ This is not the way the definition appears in $[\mathbf{D M}]$ but the two definitions agree. Given a category fibered in groupoids $\mathcal{M}, \operatorname{Hom}(\mathrm{e} / X, \mathcal{M})$ defines the value of the associated strict presheaf of groupoids on $\mathcal{C}$ at $X[\mathbf{H}]$.
} 
The first adjunction generalizes to give a Quillen pair for diagrams of simplicial sets:

$$
B: s \operatorname{Set}^{I^{o p}} \leftrightarrow s \operatorname{Set} / N I: \Gamma,
$$

where $B F$ denotes the coend of the $I$ diagrams $N(I /-) \otimes_{I} F$, which is the standard model for the homotopy colimit. This adjunction is a homotopical surjection (in the sense of Dugger [Dg, Definition 6.10]) expressing $s$ Set/NI as the localization of $s \mathcal{S} e t^{I^{o p}}$ consisting of $I$-diagrams with all maps weak equivalences (see Corollary 2.12). When $I$ is a groupoid $(B, \Gamma)$ is a Quillen equivalence (Theorem 2.7).

When $I$ is a groupoid the second adjunction also generalizes to a Quillen equivalence (Proposition 3.5):

$$
p: s \mathcal{S e t} / N I \leftrightarrow s \operatorname{Set}^{I^{o p}}: B,
$$

which is exactly the adjunction used by Dwyer, Farjoun, and Kan [DDK] to prove that the homotopy theories of $G-s \mathcal{S e t}$ (with $G$ a group and weak equivalences those on the underlying simplicial sets) and $s$ Set/BG are equivalent.

We then proceed to generalize the above constructions for diagrams indexed on the Grothendieck construction of a functor $G: I^{o p} \rightarrow$ Cat. We define an adjoint pair (Proposition 4.3)

$$
\mathcal{B}: s \operatorname{Set}^{\left(I /-\otimes_{I} G\right)^{o p}} \longrightarrow\left(s \operatorname{Set}^{I^{o p}}\right) / N G: \mathfrak{G},
$$

which form a Quillen pair from the projective model structure on the left to the injective model structure on the right. Furthermore we prove it is a Quillen equivalence when $G$ is a diagram of groupoids (Theorem 4.4). There is also a Quillen equivalence in the opposite direction $(\mathfrak{p}, \mathcal{B})$ defined only when $G$ is a diagram of groupoids and there are versions of these results for diagrams of categories and groupoids (Theorem 5.2).

In Section 5.1 we provide an interesting application of the above equivalence. We use Theorem 5.2 to prove the theorem of Dwyer and Kan [DK, Theorem 3.4] describing diagrams indexed on EI-categories (categories where all endomorphisms are isomorphisms) in terms of certain diagrams of fibrations (see Theorem 5.6).

Finally we return to our original motivation, taking $I=\mathcal{C}$ and $G=\mathcal{M}$ a presheaf of groupoids, we show that one can localize the Quillen equivalence 1 and prove that $P(\mathrm{C} / \mathcal{M}, \mathcal{G r p d})_{L}$, it the homotopy theory of stacks on $\mathcal{M}$ is Quillen equivalent to $P(\mathcal{C}, \mathcal{G r p d})_{L} / \mathcal{M}$, the homotopy theory of stacks over $\mathcal{M}$ (Theorem 6.2). We also prove the analog for presheaves of simplicial sets: namely, $P(\mathcal{C} / \mathcal{M}, s \mathcal{S} e t)_{L}$ is Quillen equivalent to $P(\mathrm{C}, s \mathrm{~S} e t)_{L} / N \mathcal{M}$.

The Quillen equivalences above generalize the equivalence of categories between sheaves on a stack $\mathcal{M}$ and fibrations in $P(\mathcal{C}, \mathcal{M})$ with target $\mathcal{M}$ and discrete fibers, proved in $[\mathbf{H 2}]$. They will be used in $[\mathbf{H 3}]$ to prove homotopical descent results for complexes of sheaves on a stack.

\subsection{Relation with other work}

Much of the work in this paper was developed as a continuation of my 2001 Ph.D. thesis. Since then a paper by Jardine $[\mathbf{J}]$ has appeared which addresses the motivating question of the paper. Though there is considerable overlap with the present paper, Jardine works with a different (though Quillen equivalent) model category and his proofs and overall approach are quite different. 


\section{Acknowledgements}

The author thanks G. Granja for many helpful discussions.

\section{The right adjoint to the Grothendieck construction}

\subsection{The coend adjunction}

We will start by describing a general adjunction involving the coend and then specialize it to the cases which interest us.

Let $\mathcal{C}$ be a closed monoidal category. We will assume that $\mathcal{C}$ is complete and cocomplete and write $*$ for the final object. Let $I$ be a small category and $G: I \rightarrow \mathcal{C}$ a functor. If $F: I^{o p} \rightarrow \mathcal{C}$ is a functor, we write

$$
G \otimes_{I} F=\operatorname{colim}\left(\coprod_{i \rightarrow j} G(i) \otimes F(j) \rightrightarrows \coprod_{i} G(i) \otimes F(i)\right)
$$

for the coend of the functors $F$ and $G$. Note that this is functorial in $F, I$ and $G$. More precisely, given $F, F^{\prime}: I \rightarrow \mathcal{C}$ and $G: I^{o p} \rightarrow \mathcal{C}$, a natural transformation $F \rightarrow F^{\prime}$ induces a map

$$
F \otimes_{I} G \rightarrow F^{\prime} \otimes_{I} G
$$

and similarly for a natural transformation $G \rightarrow G^{\prime}$. We also have functoriality with respect to the indexing category: given

$$
\iota: I \rightarrow J, \quad F: J \rightarrow \mathcal{C}, \quad G: J^{o p} \rightarrow \mathcal{C},
$$

there is a canonical map in $\mathcal{C}$

$$
(F \circ \iota) \otimes_{I}(G \circ \iota) \rightarrow F \otimes_{J} G
$$

which is induced by the map

$$
\coprod_{i \in I} F(\iota(i)) \times G(\iota(i)) \longrightarrow \coprod_{j \in J} F(j) \times G(j) .
$$

Writing $*$ for the constant functor assigning the final object, there are natural maps

$$
G(i) \rightarrow\left(G \otimes_{I} *\right)
$$

which together yield a functor

$$
\underline{G}: I \rightarrow\left(\mathcal{C} /\left(G \otimes_{I} *\right)\right) .
$$

Proposition 2.1. Let $G: I \rightarrow \mathcal{C}$ be a fixed diagram. Then

$$
\left(G \otimes_{I}-\right): \mathcal{C}^{I^{o p}} \longleftrightarrow \mathcal{C} /\left(G \otimes_{I} *\right): \operatorname{Hom}_{\mathcal{C} /\left(G \otimes_{I} *\right)}(\underline{G},-)
$$

is an adjunction.

Furthermore, if $\mathcal{C}$ is a cofibrantly generated model category satisfying the analog of SM7 and $G$ is a diagram of cofibrant objects, then the above adjunction is a Quillen pair between the projective model structure on $\mathrm{C}^{I^{o p}}$ and the usual model structure on the over category $\mathrm{C} /\left(G \otimes_{I} *\right)$. 
Proof. We compute that

$$
\begin{aligned}
& \operatorname{Hom}_{\mathcal{C} /\left(G \otimes_{I^{*}}\right)}\left(G \otimes_{I} F, C\right) \\
& \left.=\operatorname{Hom}_{\mathcal{C} /\left(G \otimes_{\left.I^{*}\right)}(\operatorname{coeq}\right.}\left(\coprod_{i \rightarrow j} G(i) \otimes F(j) \Rightarrow \coprod_{i} G(i) \otimes F(i)\right), C\right) \\
& =\operatorname{eq}\left[\prod_{i} \operatorname{Hom}_{\mathcal{C} /\left(G \otimes_{\left.I^{*}\right)}\right.}(G(i) \otimes F(i), C) \Rightarrow \prod_{i \rightarrow j} \operatorname{Hom}_{\mathcal{C} /\left(G \otimes_{I^{*}}\right)}(G(i) \otimes F(j), C)\right] \\
& =\operatorname{eq}\left[\prod_{i} \operatorname{Hom}_{\mathcal{C}}\left(F(i),\left(\mathcal{C} /\left(G \otimes_{I} *\right)\right)(G(i), C)\right)\right. \\
& \left.\quad \Rightarrow \prod_{i \rightarrow j} \operatorname{Hom}_{\mathcal{C}}\left(F(j),\left(\mathcal{C} /\left(G \otimes_{I} *\right)\right)(G(i), C)\right)\right] \\
& =\operatorname{Hom}_{\mathcal{C}^{\text {Iop }}}\left(F,\left(\mathcal{C} /\left(G \otimes_{I^{*}} *\right)\right)(\underline{G}, C)\right) .
\end{aligned}
$$

If $\mathcal{C}$ is a model category satisfying the assumptions of the statement, then the right adjoint clearly preserves fibrations and trivial fibrations.

Example 2.2. If $G$ is constant equal to the unit of the monoidal structure then

$$
G \otimes_{I} F=\operatorname{colim}_{I} F
$$

and the above adjunction is the usual adjunction between the colimit and the diagonal.

Example 2.3. Let $\mathcal{C}$ be abelian groups $\mathcal{A} b$ with the tensor product and $I$ be a group. Then a functor $I \rightarrow \mathcal{C}$ is a left $\mathbb{Z}[I]$-module $M$. Similarly functors $I^{o p} \rightarrow \mathcal{C}$ are right $\mathbb{Z}[I]$-modules. The adjunction of Proposition 2.1 is

$$
\left(-\otimes_{\mathbb{Z}[I]} M\right): \operatorname{Mod}-\mathbb{Z}[I] \longleftrightarrow \mathcal{A} b: \operatorname{Hom}_{\mathbb{Z}}(\mathbb{Z}[I],-) .
$$

Remark 2.4. One can also fix a contravariant functor $G$ and consider the coend $-\otimes_{I} G$ with covariant functors. We write our formulas for contravariant functors because we are interested in presheaves.

\subsection{The adjoint pair $(B, \Gamma)$}

Suppose now that $\mathcal{C}$ is a category admitting a map from $\mathcal{C}$ at. The examples of interest to us are Cat itself with the identity functor, Grpd via the localization functor $l:$ Cat $\rightarrow$ Grpd and $s \mathcal{S} e t$ via the nerve functor $N:$ Cat $\rightarrow$ sSet.

Applying the functor $\mathcal{C} a t \rightarrow \mathcal{C}$ to an $I$-diagram in $\mathcal{C}$ at we obtain an $I$-diagram in $\mathcal{C}$ and can then apply Proposition 2.1. The main example we are interested in is the diagram $I \rightarrow$ Cat of over categories

$$
i \mapsto I / i .
$$

When $\mathcal{C}=s$ Set or $\mathcal{G}$ rpd we will often abuse notation and omit the functor $\mathcal{C} a t \rightarrow \mathcal{C}$, writing $I$ for $N I$ or $l I$ and $I /$ - for the diagram sending

$$
\begin{aligned}
& i \mapsto N(I / i) \in s \mathcal{S e t}^{I} \\
& i \mapsto l(I / i) \in \mathcal{G r p d}^{I} .
\end{aligned}
$$


Definition 2.5. For $\mathcal{C}=\mathcal{C} a t, \mathcal{G r p d}$, or $s \mathcal{S} e t$, let $B$ denote the functor

$$
\begin{aligned}
\mathrm{C}^{I^{o p}} & \rightarrow \mathcal{C} / I \\
F & \mapsto(I /-) \otimes_{I} F,
\end{aligned}
$$

and let $\Gamma$ denote the functor

$$
\begin{gathered}
\mathcal{C} / I \rightarrow \mathcal{C}^{I^{o p}} \\
X \rightarrow I \mapsto \operatorname{Hom}_{\mathcal{C} / I}(I /-, X) .
\end{gathered}
$$

For $\mathcal{C}=\mathrm{C} a t, B F=(I /-) \otimes_{I} F$ is the category with

- objects the pairs $(i, a), a \in F(i)$, and

- morphisms $(i, a) \rightarrow(j, b)$, pairs $(\alpha, \phi)$ with $i \stackrel{\alpha}{\longrightarrow} j$ and $a \stackrel{\phi}{\longrightarrow} \alpha^{*} b \in F(i)$.

This is usually called the Grothendieck construction on $F$.

When $\mathrm{C}=s$ Set,

$$
B F=N(I /-) \otimes_{I} F
$$

is the standard model for the homotopy colimit of the diagram $F$ : it is the geometric realization of the simplicial replacement of $F$ (see $[\mathbf{B K}]$ ).

Remark 2.6. Another situation in which one can obtain a $(B, \Gamma)$-type adjunction is when one has a category $\mathcal{C}$ tensored and cotensored over $\mathcal{C}$ at. $\mathcal{C}$ could be, for instance, $s S e t^{J}$ for some indexing category $J$ with categories acting via the nerve functor.

Writing $*$ for the final object of $\mathcal{C}$, we can define $B: \mathcal{C}^{I^{o p}} \rightarrow \mathcal{C} /(I \otimes *)$ in the obvious way, and then the right adjoint $\Gamma: \mathcal{C} /(I \otimes *) \rightarrow \mathcal{C}^{I^{o p}}$ can be defined by the pullback

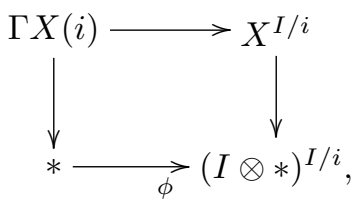

where $\phi$ is the adjoint to the map $(I / i \otimes *) \rightarrow(I \otimes *)$.

\subsection{Model structures}

Recall that Cat has a cofibrantly generated, closed simplicial model category structure in which

- weak equivalences are equivalences of categories,

- cofibrations are functors which are inclusions on objects,

- fibrations are functors which satisfy the right lifting property with respect to the inclusion $* \hookrightarrow(* \cong *)$ (where the target category here is the category with two objects and a unique isomorphism between them).

Moreover these definitions restrict to give a model structure on Grpd (see [H, H2]) for instance. It follows that there are projective model structures on $\mathrm{C} a t^{I^{o p}}$ and on $\mathcal{G r p d} d^{I^{o p}}$, denoted $\left(\mathcal{C} a t^{I^{o p}}\right)_{\text {proj }}\left(\right.$ resp. $\left(\mathcal{G r p d} d^{I^{o p}}\right)_{\text {proj }}$ ) where weak equivalences and fibrations are levelwise (see $[\mathbf{H i}]$ ). So for $\mathcal{C}=\mathcal{C} a t, \mathcal{G} r p d$, or $s \mathcal{S} e t$ Proposition 2.1 implies that $(B, \Gamma)$ is a Quillen pair. 
In this case, there are also injective model structures on $\mathrm{C}^{I^{o p}}$ where weak equivalences and cofibrations are levelwise $[\mathbf{H e}]$. Since $B$ preserves levelwise cofibrations for each of these values of $\mathcal{C},(B, \Gamma)$ is also a Quillen pair with source $\mathfrak{C}_{i n j}^{I^{o p}}$.

The main result of this section is the following.

Theorem 2.7. If $\mathcal{C}=\mathcal{C} a t, \mathcal{G r p d}$, or sSet, and $I$ is a groupoid, then the pair

$$
B: \mathrm{C}^{I^{o p}} \longleftrightarrow \mathcal{C} / N I: \Gamma
$$

is a Quillen equivalence between the projective model structure on diagrams and the over category model structure.

The previous result is not formal as the following example shows.

Example 2.8. Let $\mathcal{C}=s \mathcal{A} b$. Given $F \in \mathcal{C}^{I^{o p}}, B F=\mathbb{Z} \cdot N(I /-) \otimes_{I} F$. Since the final object of $\mathcal{C}$ is 0 , the target of $B$ is just simplicial abelian groups. In the case when $I$ is a group and $F$ is a simplicial $\mathbb{Z}[I]$-module, then $B F$ is the bar resolution

$$
\mathbb{Z}[E I] \otimes_{\mathbb{Z}[I]} M \in s \mathcal{A} b .
$$

Clearly the pair $(B, \Gamma)$ is not a Quillen equivalence in this case.

In the proof we will use the following observation: an $n$-simplex in $B(F)$ is represented uniquely by a pair

$$
\left(i_{0} \rightarrow \cdots \rightarrow i_{n}=i_{n}, x\right) \in N\left(I / i_{n}\right)_{n} \times F\left(i_{n}\right)_{n} .
$$

Abbreviating $i_{0} \rightarrow \cdots \rightarrow i_{n}=i_{n}$ by $\left(i_{0}, \ldots, i_{n}\right)$ and writing $\alpha: i_{n-1} \rightarrow i_{n}$ for the last map in the sequence, the boundary maps are given by the formulas

$$
d_{l}\left(i_{0}, \cdots, i_{n}, x\right)= \begin{cases}\left(i_{0}, \ldots, \widehat{i_{l}}, \ldots, i_{n}, d_{l}(x)\right) \in N\left(I / i_{n}\right) \times F\left(i_{n}\right) & \text { if } 0 \leqslant l<n \\ \left(i_{0}, \ldots, i_{n-1}, d_{n} \alpha^{*}(x)\right) \in N\left(I / i_{n-1}\right) \times F\left(i_{n-1}\right) & \text { if } l=n .\end{cases}
$$

Since $B(F)$ is a model for the homotopy colimit of a diagram it follows that $B$ sends levelwise weak equivalences of diagrams to weak equivalences of simplicial sets.

Proof of Theorem 2.7. We will check that the derived unit and counit are weak equivalences. Note that for $\mathcal{C}=s \mathcal{S} e t$, $\mathrm{C} a t, \mathcal{G} r p d$, the functor $B$ preserves weak equivalences and hence it is its own derived functor.

We will start by showing that $B$ takes fibrant objects to fibrant objects. This will reduce our problem to showing that the unit and counit are weak equivalences on fibrant objects. In fact, if $F \rightarrow F^{\prime}$ is a fibrant replacement, then

$$
F \rightarrow F^{\prime} \rightarrow \Gamma B\left(F^{\prime}\right)
$$

will be the derived unit of $F$. The analogous statement for the counit is an immediate consequence of the fact that $B$ is its own derived functor. 
If $\mathcal{C}=$ Cat or $\mathrm{Grpd}$ it is clear from the definition that $B$ takes fibrations to fibrations. When $\mathrm{C}=s \mathcal{S} e$, we have to produce a lift in the diagram

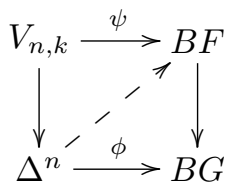

for each $k=0, \ldots, n$. Let $\phi$ be the simplex

$$
i_{0} \rightarrow \cdots \rightarrow i_{n-1} \stackrel{\alpha}{\rightarrow} i_{n}, \phi \in G\left(i_{n}\right)_{n} .
$$

If the maps in $F$ are isomorphisms, then formula (3) for the boundaries of simplices in $B F$ shows that giving a horn in $B F$ is equivalent to giving a horn in $F\left(i_{n}\right)$ over $\phi \in G\left(i_{n}\right)_{n}$ (or in any of the $F\left(i_{k}\right)$ 's). It follows that the lift exists since $F\left(i_{n}\right) \rightarrow G\left(i_{n}\right)$ is a fibration.

Writing $\eta: F \rightarrow \Gamma B F$ for the unit, evaluation at $i$ yields a commutative diagram

$$
F(i) \underset{=}{\stackrel{\eta(i)}{\longrightarrow} \Gamma(B F)(i) \stackrel{e v}{\longrightarrow}} F(i) .
$$

When $I$ is a groupoid, $\{i\} \rightarrow N(I / i)$ (respectively $\{i\} \rightarrow I / i)$ is a trivial cofibration and so, if $F$ is fibrant, the map $\Gamma(B F)(i) \rightarrow F(i)$ is a weak equivalence. It follows that $\eta(i)$ is also a weak equivalence.

Let $\mathrm{C}=s$ Set. If $X \rightarrow N I$ is a fibration, then $B \Gamma X \rightarrow N I$ is also a fibration so it suffices to see that the counit

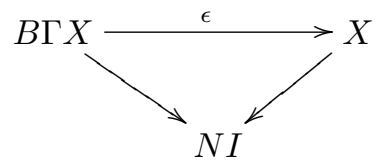

is a weak equivalence when restricted to the fibers. The fiber of $B \Gamma X \rightarrow N I$ over a vertex $i$ is $\Gamma(X)(i)=\operatorname{Map}_{N I}(N(i / I), X)$ and the restriction of the counit to the fiber is evaluation at $\{i\}$ which concludes the proof for $\mathcal{C}=s \mathcal{S} e t$.

If $\mathcal{C}=\mathcal{C}$ at or $\mathcal{G r p d}$, then the same logic applies, since it is easy to check that given a diagram of categories

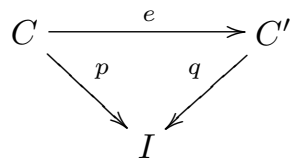

with $p$ and $q$ fibrations and $I$ a groupoid, the map $e$ is an equivalence of categories if and only if it is when restricted to the fibers over each object of $I$.

Example 2.9. Let $\pi: G \rightarrow H$ be a surjective group homomorphism regarded as an object in $\operatorname{Grpd} / H$. Let $K \subset G$ be the kernel of $\pi$. The groupoid $\Gamma(G \stackrel{\pi}{\rightarrow} H)$ has objects the set theoretic sections of $\pi$ over $H-\{1\}$, i.e., collections

$$
\left(g_{h}\right)_{h \in H \backslash\{1\}} \mid g_{h} \in G, \pi\left(g_{h}\right)=h,
$$


and isomorphisms $\left(g_{h}\right) \stackrel{\left(k_{h}\right)}{\longrightarrow}\left(g_{h}^{\prime}\right)$ are $K$-valued functions on $H$

$$
\left(k_{h}\right)_{h \in H}, k_{h} \in K, \quad g_{h}^{\prime}=k_{1} g_{h} k_{h}^{-1},
$$

where the composition is given by pointwise multiplication of these $K$-valued functions. The map $\Gamma(G \stackrel{\pi}{\longrightarrow} H) \rightarrow H$ is evaluation at $1 \in H$.

There is a (right) action of $H$ on the category $\Gamma(G \stackrel{\pi}{\longrightarrow} H)$ given on objects by

$$
a^{*}\left(g_{h}\right)=\left(g_{a^{-1}}^{-1} g_{a^{-1} h}\right)_{h}, \quad h \in H \backslash\{1\}
$$

and on isomorphisms by precomposition.

The groupoid $B \Gamma(G \stackrel{\pi}{\longrightarrow} H)$ has the same objects as $\Gamma(G \stackrel{\pi}{\rightarrow} H)$ and isomorphisms

$$
\left(a,\left(k_{h}\right)_{h \in H}\right):\left(g_{h}\right) \rightarrow\left(a^{-1}\right)^{*}\left(\left(k_{1} g_{h} k_{h}^{-1}\right)_{h}\right)=\left(k_{a} g_{a}^{-1} g_{a h} k_{a h}^{-1}\right)_{h} .
$$

The counit

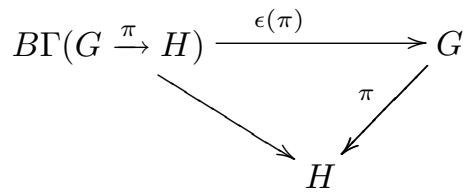

sends an isomorphism

$$
\left(\left(g_{h}\right) \stackrel{\left(a,\left(k_{h}\right)_{h \in H}\right)}{\longrightarrow}\left(k_{a} g_{a}^{-1} g_{a h} k_{a h}^{-1}\right)_{h}\right) \mapsto g_{a} k_{1} .
$$

\subsection{More about $(B, \Gamma)$ for simplicial sets}

Proposition 2.10. If $Y \rightarrow N I$ is a fibration, then the counit $B \Gamma Y \rightarrow Y$ is a weak equivalence. Equivalently, the derived counit $B \underline{\Gamma} Z \rightarrow Z$ is always a weak equivalence.

Proof. The counit is given by the evaluation map

$$
B \Gamma Y=N(I /-) \otimes_{I} \operatorname{Map}_{N I}(N(I /-), Y) \rightarrow Y .
$$

The induced map on the fibers over $i \in N I$ is the map

$$
\Gamma Y(i)=\operatorname{Map}_{N I}(N(I / i), Y) \rightarrow \operatorname{Map}_{N I}(\{i\}, Y)
$$

which is a weak equivalence since $Y \rightarrow N I$ is a fibration.

Since $\Gamma Y$ is a diagram of weak equivalences, a version of Quillen's theorem B (see [GJ, Lemma 5.7, p. 237]) shows that the fibers of the map $B \Gamma Y \rightarrow N I$ are weakly equivalent to the homotopy fibers and it then follows that the counit is a weak equivalence.

Proposition 2.11. The derived unit $F \rightarrow \underline{\Gamma B F}$ is a weak equivalence if and only if $F$ is a diagram sending each arrow in $I$ to a weak equivalence.

Proof. Again by Quillen's theorem B (or more precisely [GJ, Lemma 5.7, p. 237]), $B F \rightarrow N I$ is a quasi-fibration (i.e. the fibers agree with the homotopy fibers) and hence the restriction of a fibrant replacement

$$
B F \rightarrow \overline{B F}
$$

to the fiber over $i$ is a weak equivalence. Hence the unit

$$
F(i)=B F_{\mid i} \rightarrow \overline{B F}_{\mid i} \simeq \operatorname{Map}_{N I}(N(I / i), \overline{B F})=\underline{\Gamma} B F
$$


is also a weak equivalence. The converse is clear.

Corollary 2.12. The adjoint pair $(B, \Gamma)$ induces a Quillen equivalence from the localization of $s \mathcal{S} e t_{\text {proj }}^{I^{o p}}$ with respect to all maps between representable functors to sSet/NI.

Proof. Proposition 2.10 says that the derived counit is a weak equivalence. Proposition 2.11 tells us that for local objects in $s \mathcal{S}_{e} t^{I^{o p}}$ the derived unit is a weak equivalence, which completes the proof.

Remark 2.13. Proposition 2.10 says that the Quillen map $(B, \Gamma): s \mathcal{S}_{e} t_{\text {proj }}^{I^{o p}} \rightarrow s \mathcal{S} e t / N I$ is homotopically surjective in the sense of $[\mathbf{D g}$, Definition 6.10] and so, by [Dg, Proposition 6.11], $(B, \Gamma)$ induces a Quillen equivalence from a localization of $s \mathcal{S} e t_{p r o j}^{I^{o p}}$ to $s \mathcal{S} e t / N I$.

Remark 2.14. One can show that in fact the unit (not the derived unit) is a weak equivalence for any diagram because in fact the canonical map $\operatorname{set}(N(I / i), F(i)) \rightarrow$ $\Gamma B F(i)$ is an isomorphism as every simplex in $B F(i)$ is the boundary of a simplex ending at $i$.

Using this observation we can prove a relative version of Proposition 2.11 and see that if $F \in s \mathcal{S} e t^{I^{o p}}$ is a diagram of fibrant simplicial sets and we factor $B G \rightarrow B F$ into a trivial cofibration followed by a fibration $B G \rightarrow \overline{B G} \rightarrow B F$, then $G \rightarrow \Gamma \overline{B G} \times_{\Gamma B F} F$ is a weak equivalence if and only if for each map $\alpha: i \rightarrow j \in I$, the square

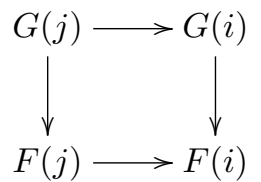

is homotopy cartesian.

The assignment $i \rightarrow s \mathcal{S e t} / F(i)$ is an $I$-diagram of categories under pullback. The end of this $I$-diagram

$$
\operatorname{End}\left((\operatorname{set} / F(i))^{I / j}\right)=\mathrm{eq}\left(\prod_{i \in I}(\operatorname{set} / F(i))^{I / i} \Rightarrow \prod_{i \rightarrow j \in I}(\operatorname{set} / F(i))^{I / j}\right)
$$

is the category $s \mathcal{S} e t^{I^{o p}} / F$, and so we have that $\Gamma$ induces a functor from $s \mathcal{S} e t / B F$ to $\operatorname{End}\left((s \mathcal{S} e t / F(i))^{I / j}\right)$.

In an appropriate sense this is writing $s \mathcal{S} e t / B F$ as the homotopy limit of the model categories $s$ Set $/ F(i)$. More precisely, the diagram

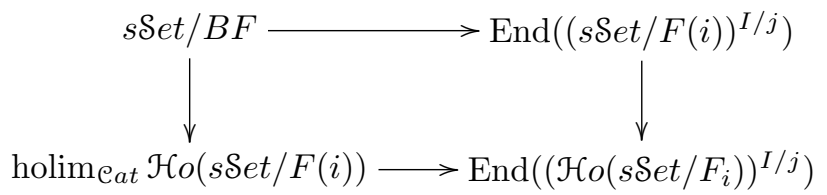

induces an equivalence of categories between $\mathcal{H} o(s \mathcal{S} e t / B F)$ and the homotopy category of the pullback of the other three categories. 


\subsection{Application to fibered categories}

A fibered category $\mathcal{D} \stackrel{\pi}{\longrightarrow} \mathcal{C}$ is a functor such that for each $d \in \mathcal{D}$ and $\alpha: c \rightarrow \pi(d) \in$ $\mathcal{C}$, the over category $(\mathcal{D} / d) \downarrow \alpha$ has a final object projecting to $\alpha$ in $\mathcal{C} / \pi(d)$ (it is easy to prove that this agrees with the original definition $[\mathbf{G i}]$ ).

As an application of the adjunction $(B, \Gamma)$ discussed above we will now show that fibered categories over $\mathcal{C}$ are precisely those which, as categories over $\mathcal{C}$, are equivalent to categories of the form $B G \rightarrow \mathcal{C}$ for some functor $G: \mathcal{C}^{o p} \rightarrow \mathcal{C} a t$.

Lemma 2.15. Let $\mathcal{D} \stackrel{\pi}{\longrightarrow} \mathcal{C}$ be a fibered category. Then

(a) For $\epsilon: B \Gamma \mathcal{D} \rightarrow \mathcal{D}$ the counit of the adjunction $(B, \Gamma)$ and $d \in \mathcal{D}$, the over category $\epsilon \downarrow d$ has a final object

$$
\left((\pi(d), s(d): \mathcal{C} / \pi(d) \rightarrow \mathcal{D}), \operatorname{id}_{d}\right) .
$$

(b) The counit $\epsilon: B \Gamma \mathcal{D} \rightarrow \mathcal{D}$ determines a bijection

$$
\operatorname{Hom}_{B \Gamma \mathcal{D}}\left(\left(\pi\left(d_{1}\right), s\left(d_{1}\right)\right),\left(\pi\left(d_{2}\right), s\left(d_{2}\right)\right)\right) \rightarrow \mathcal{D}\left(d_{1}, d_{2}\right) .
$$

Proof.

(a) For each object $\alpha: c \rightarrow \pi(d)$ of $\mathcal{C} / \pi(d)$ we can choose a final object $\tilde{\alpha}: \tilde{c} \rightarrow d$ in $(\mathcal{D} / d) \downarrow \alpha$ with $\pi(\tilde{\alpha})=\alpha$. We make this choice so that if $\alpha=\operatorname{id}_{\pi(d)}$ then $\tilde{\alpha}=\operatorname{id}_{d}$. We define

$$
s(d)(\alpha)=\tilde{c} .
$$

The universal property of $\tilde{\alpha}$ implies that this assignment on objects extends to a functor

$$
s(d): \mathcal{C} / \pi(d) \rightarrow \mathcal{D}
$$

over $\mathcal{C}$, and hence an object in $\Gamma \mathcal{D}(\pi(d)) .(\pi(d), s(d))$ is then an object in $B \Gamma(\pi(d))$ and by our choice of lift of $\operatorname{id}_{\pi(d)}$, we have that $\epsilon(\pi(d), s(d))=d$. Thus $((\pi(d), s(d)), d \stackrel{=}{\longrightarrow} d)$ is a well-defined object in $\epsilon \downarrow d$ which we must show is final. Let $(c, s, \beta: s(c=c) \rightarrow d)$ be another object in $\epsilon \downarrow d$. If

$$
(\alpha, \phi):(c, s, \beta) \rightarrow(\pi(d), s(d), d=d)
$$

is a map in $\epsilon \downarrow d$ then the diagram

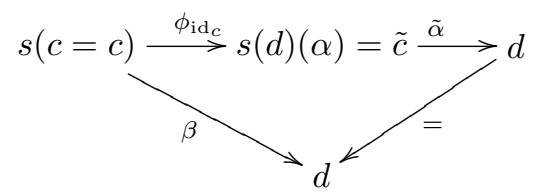

commutes, as $\tilde{\alpha}$ is the image of the map $\alpha: \alpha \rightarrow \mathrm{id}_{d} \in \mathcal{D} / d$ under $s(d)$. It follows that $\alpha=\pi(\beta)$ and $\phi_{\mathrm{id}_{c}}$ is necessarily the canonical map.

Given $\gamma: c^{\prime} \rightarrow c$, the commutativity of the diagram

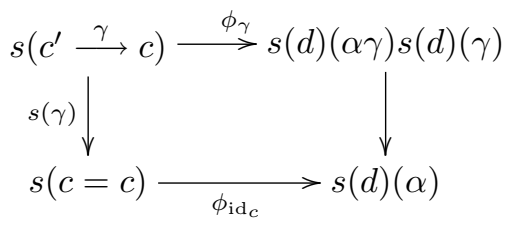


shows that there is a unique choice for $\phi(\gamma)$, as $s(d)(\gamma)$ is a final object in $(\mathcal{D} / \tilde{c}) / \gamma$. Thus there is at most one map $(c, s, \beta) \rightarrow\left(\pi(d), s(d), \mathrm{id}_{d}\right)$ and it is easy to check that the canonical choices described above constitute such a map.

(b) Consider a map $(\alpha, \phi):\left(\pi\left(d_{1}\right), s\left(d_{1}\right)\right) \rightarrow\left(\pi\left(d_{2}\right), s\left(d_{2}\right)\right)$. For each $\gamma: c \rightarrow \pi\left(d_{1}\right)$ we have a commutative diagram

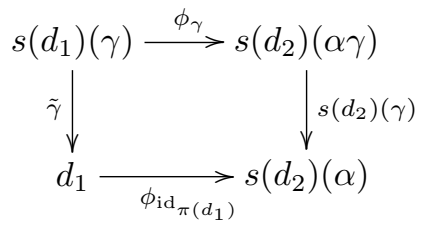

and the same argument as before shows that $\phi$ is completely determined by $\phi_{\mathrm{id}_{\pi\left(d_{1}\right)}}$. The counit assigns to the pair $(\alpha, \phi)$ the composite

$$
d_{1} \stackrel{\phi_{\mathrm{id}_{\pi\left(d_{1}\right)}}^{\longrightarrow}}{\longrightarrow} \tilde{c} \stackrel{\tilde{\alpha}}{\longrightarrow} d_{2}
$$

with $\tilde{\alpha}$ the final object in $\left(\mathcal{D} / d_{2}\right) \downarrow \alpha$. The universal property of $\tilde{\alpha}$ easily implies that the counit is a bijection.

Proposition 2.16. A functor $\mathcal{D} \stackrel{\pi}{\longrightarrow} \mathcal{C}$ is a fibered category if and only if it is equivalent in $\mathcal{C}$ at/ $/ \mathcal{C}$ to $B G$ for some functor $G$ : $\mathcal{C}^{o p} \rightarrow$ Cat.

Proof. Using the notation of Lemma 2.15, define an assignment $S: \mathcal{D} \rightarrow B \Gamma \mathcal{D}$ on objects by

$$
d \mapsto(\pi(d), s(d)) .
$$

Lemma 2.15 (b) shows that $S$ extends to a functor with image a full subcategory of $B \Gamma \mathcal{D}$. Let $\mathcal{D}^{\prime} \subset B \Gamma \mathcal{D}$ denote the strictly full subcategory determined by the image of $S$ (i.e. the full subcategory whose objects are all those isomorphic to an object in the image of $S$ ).

Given $\alpha: c \rightarrow \pi(d)$, there is a canonical natural isomorphism $\alpha^{*} s(d) \rightarrow s(\tilde{c})$. This implies that if $(b, s) \in \mathcal{D}^{\prime}$ and $\alpha: a \rightarrow b$ is a map, then $\left(a, \alpha^{*}(s)\right)$ is also in $\mathcal{D}^{\prime}$; therefore, if we define $G(c)$ to be the fiber over $c$ of the projection $\mathcal{D}^{\prime} \rightarrow \mathcal{C}, G$ is a functor on $\mathcal{C}$ and

$$
\mathcal{D}^{\prime}=B G
$$

This concludes the proof.

\section{The left adjoint to the Grothendieck construction}

\subsection{The adjunction}

It is not very surprising that the functor $B$ discussed above admits a right adjoint. It is more surprising that in some situations it also admits a left adjoint.

Definition 3.1. Let $p: \mathcal{C} a t / I \rightarrow \mathcal{C} a t^{I^{o p}}$ be the functor defined by

$$
p(C \stackrel{\pi}{\rightarrow} I)(i)=i / I \times_{I} C .
$$


Notice that $i / I \times{ }_{I} C$ is isomorphic to the undercategory $i / \pi$ whose objects are pairs $(c, i \stackrel{\alpha}{\rightarrow} \pi(c))$ and the morphisms

$$
(c, i \stackrel{\alpha}{\longrightarrow} \pi(c)) \stackrel{\phi}{\longrightarrow}\left(c^{\prime}, i \stackrel{\alpha^{\prime}}{\longrightarrow} \pi\left(c^{\prime}\right)\right)
$$

are maps $\phi \in C$ so that the triangle

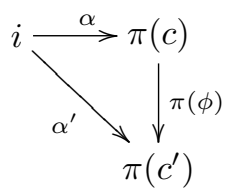

commutes.

Proposition 3.2. There is an adjoint pair of functors $p:$ Cat $/ I \longrightarrow$ Cat ${ }^{I^{o p}}: B$.

Proof. We will give a unit and a counit for the adjunction and check that they satisfy the required identities. Let $F \in \mathcal{C} a t^{I^{o p}}$. Given $\alpha: i \rightarrow j \in I$, we will write $\alpha^{*}$ for $F(\alpha)$.

The category $p(B F)(i)$ is

$$
\begin{aligned}
p B F(i) & =i / I \times_{I}\left((I /-) \otimes_{I} F\right) \\
& =\operatorname{coeq}\left[\coprod_{j \rightarrow k} i / I \times_{I}(I / j \times F(k)) \Rightarrow \coprod_{j} i / I \times_{I}(I / j \times F(j))\right] .
\end{aligned}
$$

The counit is the canonical evaluation map from the previous coequalizer to the coequalizer of

$$
\coprod_{j \rightarrow k} \operatorname{Hom}(i, j) \times F(k) \Rightarrow \operatorname{Hom}(i, j) \times F(j),
$$

which is $\operatorname{Hom}(i,-) \otimes_{I} F=\left.\operatorname{colim}_{i / I^{o p}} F\right|_{i / I^{o p}}=F(i)$. Explicitly, $p(B F)(i)$ is the category of triples $((j, b), i \stackrel{\alpha}{\rightarrow} j)$ and the counit $p B F(i) \stackrel{\epsilon_{i}}{\longrightarrow} F(i)$ sends

$$
((j, b), i \stackrel{\alpha}{\longrightarrow} j) \mapsto \alpha^{*}(b) .
$$

Given $C \stackrel{\pi}{\rightarrow} I$,

$$
B p C=(I /-) \otimes_{I}\left((-/ I) \times_{I} C\right) \cong\left((I /-) \otimes_{I}(-/ I)\right) \times{ }_{I} C .
$$

Notice that $(I /-) \otimes_{I}(-/ I)$ is the category of arrows in $I$, which we denote $P I$. The unit is the map induced by the functor $I \rightarrow P I$ which sends $i$ to its identity morphism.

Explicitly, $B p C$ is the category of pairs $(c, i \stackrel{\alpha}{\longrightarrow} \pi(c))$ with $c \in C$ and $\alpha \in \operatorname{mor}(I)$ and the unit sends $c \mapsto\left(c, i d_{\pi(c)}\right)$.

We need to check that for each $F \in \mathcal{C} a t^{I^{o p}}$, the composite

$$
B F \stackrel{\eta_{B F}}{\longrightarrow} B p B F \stackrel{B \epsilon_{F}}{\longrightarrow} B F
$$

is the identity. We will do this just for objects: $B p B F(i)$ is the category with objects $((j, b), i \stackrel{\alpha}{\longrightarrow} j)$. Now $B F \stackrel{\eta_{B F}}{\longrightarrow} B p B F$ sends $(j, b) \mapsto((j, b), j \stackrel{\equiv}{\longrightarrow} j)$ and $B p B F \stackrel{B \epsilon_{F}}{\longrightarrow}$ $B F$ sends $((j, b), i \stackrel{\alpha}{\rightarrow} j) \mapsto\left(i, \alpha^{*} b\right)$. 
Finally, we need to check that for each $C \stackrel{\pi}{\longrightarrow} I$, the composition

$$
p C \stackrel{p \eta_{C}}{\longrightarrow} p B p C \stackrel{\epsilon_{p C}}{\longrightarrow} p C
$$

is the identity. Again we just do this for objects: $p B p C$ has objects $(c, i \stackrel{\alpha}{\longrightarrow} j \stackrel{\beta}{\longrightarrow} \pi(c))$.

The functor $p C \stackrel{p \eta_{C}}{\longrightarrow} p B p C$ sends

$$
(c, i \stackrel{\alpha}{\longrightarrow} \pi(c)) \mapsto(c, i \stackrel{\alpha}{\longrightarrow} \pi(c) \stackrel{=}{\longrightarrow} \pi(c))
$$

and $p B p C \stackrel{\epsilon_{p C}}{\longrightarrow} p C$ sends

$$
(c, i \stackrel{\alpha}{\longrightarrow} j \stackrel{\beta}{\longrightarrow} \pi(c)) \mapsto(c, i \stackrel{\beta \circ \alpha}{\longrightarrow} \pi(c)) .
$$

Clearly the previous result also holds (with the same proof) if we replace $C a t$ with Grpd, as long as $I$ is a groupoid. The analogous adjunction for simplicial sets works only when $I$ is a groupoid. We can again define a functor $p$ as before.

Definition 3.3. Let $p: s \operatorname{Set} / N I \rightarrow s \operatorname{Set}^{I^{o p}}$ be the functor defined by

$$
p X(i)=N(i / I) \times_{N I} X .
$$

However, this will be the left adjoint to $B$ only when $I$ is a groupoid. The explanation is contained in the following lemma.

Lemma 3.4. If $I$ is a groupoid, then the canonical map

$$
N(I /-) \otimes_{I} N(-/ I) \rightarrow N\left((I /-) \otimes_{I}(-/ I)\right)
$$

is an isomorphism.

Proof. First note that $(I /-) \otimes_{I}(-/ I)$ is the Grothendieck construction on the functor $i \mapsto i / I$ and this is easily identified with the category of arrows in $I$. An $n$-simplex in $N(I /-) \otimes_{I} N(-/ I)$ is represented uniquely (see (2)) by

$$
\left(i_{0} \rightarrow \cdots \rightarrow i_{n}, i_{n} \rightarrow i_{n+1} \rightarrow i_{2 n+1}\right) \in N(I) \times N\left(i_{n} / I\right) .
$$

The natural map sends such an $n$-simplex to the $n$-simplex

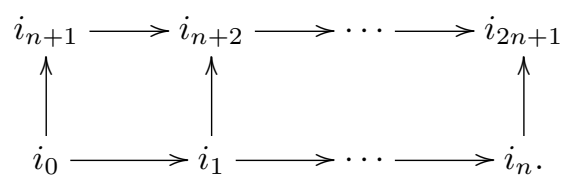

Clearly this is an isomorphism if $I$ is a groupoid.

Proposition 3.5. If $I$ is a groupoid, then

$$
p: s \mathcal{S} e t / N I \Leftrightarrow\left(s \mathcal{S} e t^{I^{o p}}\right)_{p r o j}: B
$$

is an adjoint pair.

Proof. The counit of the adjunction can be defined exactly as in the proof of Proposition 3.2 (whether $I$ is a groupoid or not). Using Lemma 3.4, the unit can also be defined analogously when $I$ is a groupoid and one then checks, as in Proposition 3.2, that the required identities are satisfied. 
Remark 3.6. $B$ has a left adjoint $q$ when $I$ is an arbitrary indexing category. This is in general different from the obvious generalization of the functor $p$ defined above (but does agree if $I$ is a groupoid). Using the description of the simplices of $B F$ before the statement of Theorem 2.7, it is not hard to check that, given $Y \stackrel{\pi}{\rightarrow} N I \in s \mathcal{S} e t / N I$, $q(Y) \in s \operatorname{Set}^{I^{o p}}$ can be defined by the formula

$$
q(Y)(i)=\operatorname{colim}_{\Delta^{k} \stackrel{\beta}{\longrightarrow} Y \in \Delta(Y)} \Delta^{k} \times I(i, \pi(\beta(k))) .
$$

It is not clear to us whether it is possible to define suitable model structures so that $(q, B)$ is a Quillen map.

\subsection{Model structures}

It is now easy to relate the adjunction above with the model categories on diagrams and the over categories.

\section{Proposition 3.7.}

(a) For I an arbitrary small category, $(p, B)$ is a Quillen map from Cat/I to $\left(\mathrm{C} a t^{I^{o p}}\right)_{\text {proj }}$.

(b) If I is a groupoid and $\mathrm{C}=\mathrm{Cat}, \mathcal{G r p d}$, or sSet, then

$$
p: \mathcal{C} / \phi(I) \longleftrightarrow\left(\mathcal{C}^{I^{o p}}\right)_{p r o j}: B
$$

is a Quillen equivalence.

Proof.

(a) $B$ sends weak equivalences to weak equivalences. Additionally, $B$ sends objectwise fibrations to fibrations since if $(i, x) \stackrel{(\alpha, f)}{\longrightarrow}\left(i^{\prime}, x^{\prime}\right)$ is an isomorphism in $B F$ then $\alpha$ is an isomorphism in $I$ and $f$ is an isomorphism in $F(i)$. This shows $(p, B)$ is a Quillen pair.

(b) Let $\mathcal{C}=$ Cat. When $I$ is a groupoid $(B, \Gamma)$ is a Quillen equivalence by Theorem 2.7. Since $B$ is its own derived functor, it induces an equivalence of homotopy categories so $(p, B)$ is also a Quillen equivalence. It is clear that $(p, B)$ restricts to a Quillen equivalence $\operatorname{Grpd} / I \longleftrightarrow\left(\mathcal{G r p d} d^{I^{o p}}\right)_{\text {proj }}$.

When $\mathcal{C}=s \mathcal{S} e t,(p, B)$ is a Quillen pair since $B$ preserves weak equivalences and fibrations (see $(4))$. Since $(B, \Gamma)$ is a Quillen equivalence and $B$ is its own derived functor, $B$ induces an equivalence on the level of homotopy categories and hence $(p, B)$ is a Quillen equivalence.

Example 3.8. The equivalence between the homotopy theory of $I$-diagrams and fibrations over $N I$ when $I$ is a group is a well-known theorem of Dwyer, Kan, and DrorFarjoun $[\mathbf{D D K}]$. Their equivalence was essentially the pair $(p, B)$ but was not stated in the language of model categories. We now describe this case in more detail.

Suppose the indexing category $I$ is a group $G$. We will adopt standard notation and write $B G$ instead of $N I$ and $E G$ for $N(I / *)$, which comes with a left $G$-action. We will write $E G^{o p}=N(* / I)$. This carries a right $G$-action. An $I^{o p}$-diagram is a simplicial 
set $X$ with a right $G$-action and the previous propositions give us two adjunctions between $s$ Set $/ B G$ and right $G$-spaces. We have

$$
B X=X \times_{G} E G \quad(\text { Borel construction) }
$$

and

$$
\Gamma(Y \rightarrow B G)=\operatorname{Map}_{B G}(E G, Y), \quad p(Y \rightarrow B G)=Y \times_{B G} E G^{o p} .
$$

In the $(B, \Gamma)$ adjunction, the counit

$$
B \Gamma Y=\operatorname{Map}_{B G}(E G, Y) \times_{G} E G \rightarrow Y
$$

is given by evaluation. It is the universal map from a $G$-bundle to $Y$ over $B G$. The unit is the $G$-equivariant map

$$
X \rightarrow \Gamma B X=\operatorname{Map}_{B G}\left(E G, X \times_{G} E G\right) \quad x \mapsto(e \mapsto[(x, e)]) .
$$

Note that this map has a functorial retraction.

In the $(p, B)$ adjunction, the unit

$$
Y \rightarrow B p Y=\left(Y \times_{B G} E G^{o p}\right) \times_{G} E G
$$

gives the universal map over $B G$ from $Y$ to a $G$-bundle. Note that by Lemma 3.4, $E G^{o p} \times_{G} E G$ is the free path space $P B G$ on $B G$ and so $B p Y=Y \times_{B G} P B G$.

The counit

$$
p B X=\left(X \times_{G} E G\right) \times{ }_{B G} E G^{o p} \rightarrow X
$$

can be identified with the map $E G \times X \rightarrow X$ with the anti-diagonal action on the domain.

\section{Diagrams indexed by a Grothendieck construction}

In this section, we will give a more sophisticated version of the adjunction in Section 2 which relates diagrams indexed on a Grothendieck construction to diagrams indexed on the original category.

We begin by recalling the naturality of a coend. Given an indexing category $I$ and $F, F^{\prime}: I \rightarrow \mathcal{C}$ and $G: I^{o p} \rightarrow \mathcal{C}$, a natural transformation $F \rightarrow F^{\prime}$ induces a map

$$
F \otimes_{I} G \rightarrow F^{\prime} \otimes_{I} G
$$

and similarly for a natural transformation $G \rightarrow G^{\prime}$.

We also have functoriality with respect to the indexing category: given

$$
\iota: I \rightarrow J, \quad F: J \rightarrow \mathcal{C}, \quad G: J^{o p} \rightarrow \mathcal{C},
$$

there is a canonical map in $\mathcal{C}$

$$
(F \circ \iota) \otimes_{I}(G \circ \iota) \rightarrow F \otimes_{J} G
$$

which is induced by the map

$$
\coprod_{i \in I} F(\iota(i)) \times G(\iota(i)) \longrightarrow \coprod_{j \in J} F(j) \times G(j) .
$$

We will also make use of the following observation: given functors $F: I^{o p} \rightarrow$ Cat and $G: B F \rightarrow$ Cat, a map $\alpha: i \rightarrow j$ determines a functor $\alpha^{*}: F(j) \rightarrow F(i)$. For each 
$(j, a) \in B F$ it also determines a map $\left(i, \alpha^{*}(a)\right) \rightarrow(j, a)$ and this yields a natural transformation

$$
G_{\mid F(j)} \stackrel{\eta_{\alpha}}{\longrightarrow} G_{\mid F(i)} \circ \alpha^{*}
$$

given by

$$
G_{\mid F(j)}(a)=G(j, a) \rightarrow G\left(i, \alpha^{*}(a)\right)=\left(G_{\mid F(i)} \circ \alpha^{*}\right)(a) .
$$

Similarly, there is a natural transformation of functors $F(j) \rightarrow$ Cat

$$
F(j) /-\stackrel{\epsilon_{\alpha}}{\longrightarrow}(F(i) /-) \circ \alpha^{*}
$$

given by

$$
F(j) / a \stackrel{\alpha^{*}}{\longrightarrow} F(i) / \alpha^{*}(a)
$$

Given a diagram $F: I^{o p} \rightarrow$ Cat, we will denote by $F^{o p}$ the diagram $i \mapsto F(i)^{o p}$.

Definition 4.1. Let $\mathcal{C}$ be a closed monoidal category. Let $I^{o p} \stackrel{F}{\longrightarrow}$ Cat be a diagram of categories, and $G:\left(B\left(F^{o p}\right)\right)^{o p} \rightarrow \mathcal{C}$ be a functor.

We define

$$
\mathcal{B}_{G}: \mathrm{e}^{B F^{o p}} \rightarrow \mathrm{e}^{I^{o p}} / \mathcal{B}_{G}(*)
$$

by

$$
\mathcal{B}_{G} H(i)=\left.\left.G\right|_{F(i)} \otimes_{F(i)} H\right|_{F(i)}
$$

with the natural projection to $\left.G\right|_{F(i)} \otimes_{F}(i) *$. The effect of $\mathcal{B} G$ on a morphism $i \stackrel{\alpha}{\longrightarrow} j$ is given by the composite

$$
\begin{array}{r}
\mathcal{B}_{G} H(j)=\left.\left.G\right|_{F(j)} \otimes_{F(j)} H\right|_{F(j)} \stackrel{\epsilon_{\alpha} \otimes \eta_{\alpha}}{\longrightarrow}\left(\left(\left.G\right|_{F(i)}\right) \circ \alpha^{*}\right) \otimes_{F(j)}\left(\left.H\right|_{F(i)} \circ \alpha^{*}\right) \\
\left.{ }_{\left(\left.G\right|_{F(i)} /-\right) \otimes_{F(i)}} H\right|_{F(i)}=\mathcal{B}_{G} H(i),
\end{array}
$$

where we have written $\alpha^{*}$ for $F(\alpha): F(j) \rightarrow F(i)$.

It is straightforward to check that the assignments above make $\mathcal{B}_{G} H$ functorial in $I$ and that $\mathcal{B}_{G}$ is itself a functor.

Our main concern will be when $\mathcal{C}=s$ Set, $\mathcal{C} a t$ or Grpd and $G:\left(B F^{o p}\right)^{o p} \rightarrow \mathcal{C}$ is determined by the functor $G:\left(B F^{o p}\right)^{o p} \rightarrow$ Cat defined by

$$
(i, a) \mapsto F(i) / a .
$$

In this case we will omit the subscript $G$ and simply write $\mathcal{B}$. Given $H \in \mathfrak{C}^{B F^{o p}}$, we have

$$
\mathcal{B} H(i)=B\left(\left.H\right|_{F(i)}\right)=\left.(F(i) /-) \otimes_{F(i)} H\right|_{F(i)} .
$$

We will now define the right adjoint to $\mathcal{B}_{G}$. Let $r_{i, a}: B F^{o p} \rightarrow$ Set be the representable functor

$$
r_{i, a}(j, b)=\operatorname{Hom}_{B F}((j, b),(i, a))=\coprod_{j \stackrel{\alpha}{\longrightarrow} i \in I} \operatorname{Hom}_{F(j)}\left(b, \alpha^{*}(a)\right) .
$$

There is a natural monoidal functor $\mathcal{S} e t \rightarrow \mathcal{C}$ sending $S \rightarrow \coprod_{s \in S} U$ where $U$ is the 
unit of the monoidal structure on $\mathcal{C}$. Abusing notation we will consider $r_{i, a}$ a functor $B F^{o p} \rightarrow \mathcal{C}$.

One can check that

$$
\mathcal{B}_{G}\left(r_{i, a}\right)(j)=\coprod_{j \stackrel{\alpha}{\longrightarrow} i} G\left(j, \alpha^{*}(a)\right)
$$

since the coend of a functor with a representable is evaluation at the representable. We will write $F_{i, a}$ for $\mathcal{B}\left(r_{i, a}\right)$. This is given by the formula

$$
F_{i, a}(j)=\coprod_{j \stackrel{\alpha}{\longrightarrow} i} F(j) / \alpha^{*}(a),
$$

with the canonical projection to $F=\mathcal{B}(*)$.

Definition 4.2. We define $\mathfrak{G}_{G}$ to be the functor $\mathrm{e}^{I^{o p}} / \mathcal{B}_{G}(*) \rightarrow \mathrm{e}^{B F^{o p}}$ given by

$$
\left(\mathfrak{G}_{G} K\right)(i, a)=\operatorname{Hom}_{\mathcal{C}^{I o p}} / \mathcal{B}_{G}(*)\left(\mathcal{B}_{G}\left(r_{i, a}\right), K\right) .
$$

In our main example (5) we will omit the subscript and write $\mathfrak{G}$ which is given by the formula

$$
\mathfrak{G} K(i, a)=\operatorname{Hom}_{\mathcal{C}^{I o p} / F}\left(F_{i, a}, K\right) .
$$

Proposition 4.3. The following is an adjoint pair

$$
\mathcal{B}_{G}: \mathrm{e}^{B F^{o p}} \leftrightarrow \mathrm{e}^{I^{o p}} / \mathcal{B}_{G}(*): \mathfrak{G}_{G}
$$

Proof. Let $H \in \mathrm{e}^{B F^{o p}}$ and $K \in \mathrm{e}^{I^{o p}} / \mathcal{B}_{G}(*)$. A morphism $H \rightarrow \mathfrak{G}_{G}(K)$ consists of compatible maps for each $(i, a) \in B F H(i, a) \rightarrow \operatorname{Hom}_{\mathcal{C}^{I^{o p}} / \mathcal{B}_{G}(*)}\left(\mathcal{B}_{G}\left(r_{i, a}\right), K\right)$.

Explicitly, this means that for each $k \rightarrow l \in I$ we have commutative diagrams

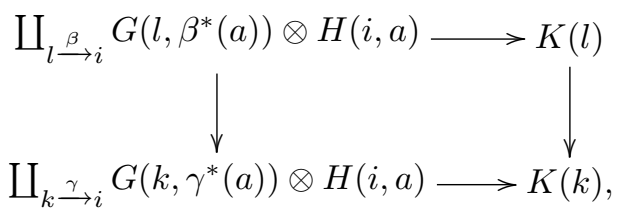

and for each $(\alpha, f):(i, a) \rightarrow(j, b) \in B F$ the following diagram commutes

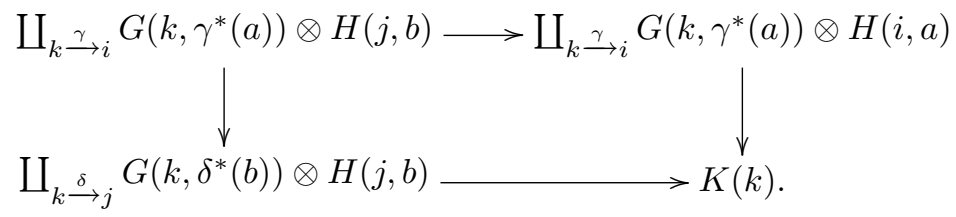

Diagram (7) for $k=j=i$ and $\alpha=$ id gives us maps

$$
\left.\left.G\right|_{F(i)} \otimes_{F(i)} H\right|_{F(i)} \rightarrow H(i)
$$

and diagram (6) together with the remaining cases of (7) provide the compatibility necessary to make this natural in $i$. Moreover one checks that the necessary conditions to get the map $\mathcal{B}_{G} H \rightarrow K$ are precisely those expressed in diagrams (6) and (7), which proves the adjunction. 
Theorem 4.4. Let $\mathcal{C}=\mathcal{C} a t$, sSet, or Grpd. Then

(a) The adjoint pair $\mathcal{B}:\left(\mathfrak{C}^{B F^{o p}}\right)_{\text {proj }} \longleftrightarrow\left(\mathfrak{C}^{I^{o p}} / F\right)_{i n j}$ : $\mathfrak{G}$ is a Quillen map.

(b) If $F$ is a diagram of groupoids, then $(\mathcal{B}, \mathfrak{G})$ is a Quillen equivalence.

Proof.

(a) In the injective model structure on these categories all objects are cofibrant. As $\mathfrak{G}$ is defined as maps out of $F_{i, a}$, it clearly sends (trivial) fibrations in $\left(\mathrm{C}^{I^{o p}} / F\right)_{i n j}$ to levelwise (trivial) fibrations.

(b) First we show that the derived counit is a weak equivalence. The counit is given by evaluation and so at an object $i \in I$ factors as

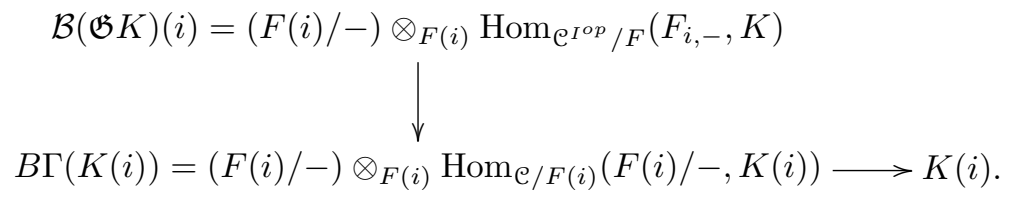

The bottom map is a weak equivalence by Theorem 2.7 and we can see that the first one is also a weak equivalence as follows: Let $r_{i}: I^{o p} \rightarrow \mathcal{S e t} \rightarrow \mathcal{C}$ be the representable functor. An element $a \in F(i)$ corresponds to a map $r_{i} \stackrel{a}{\rightarrow} F$ which we may factor as

$$
r_{i} \rightarrow r_{i} \otimes F(i) / a \rightarrow F_{i, a} \rightarrow F .
$$

Evaluated at some $j \in I$, this is

$$
\coprod_{j \stackrel{\alpha}{\longrightarrow} i} * \rightarrow \coprod_{j \stackrel{\alpha}{\longrightarrow} i} F(i) / a \rightarrow \coprod_{j \stackrel{\alpha}{\longrightarrow} i} F(j) / \alpha^{*}(a) \rightarrow F(j) .
$$

Clearly the first two maps are weak equivalences and so if $K \rightarrow F$ is a fibration,

$$
\operatorname{Hom}_{\mathcal{C}^{I^{o p}} / F}\left(F_{i, a}, K\right) \rightarrow \operatorname{Hom}_{\mathcal{C}^{I^{o p}} / F}\left(r_{i} \otimes F(i) / a, K\right) \simeq \operatorname{Hom}_{\mathcal{C} / F(i)}(F(i) / a, K(i))
$$

is a weak equivalence. This induces the vertical map in (8) so we are done with the counit.

We now check that the derived unit is a weak equivalence. It suffices to do this for $H \in \mathcal{C}^{B F^{o p}}$ levelwise fibrant. Let $R \mathcal{B} H \rightarrow F$ denote the fibrant replacement of $\mathcal{B} H \rightarrow F \in\left(\mathcal{C}^{I^{o p}} / F\right)_{i n j}$. Consider the diagram

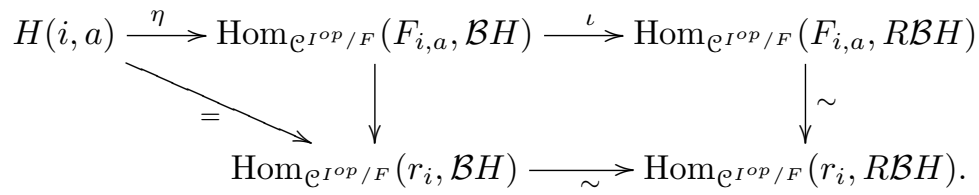

Here $\eta$ is the unit, defined by pulling back elements in $H(i, a)$. The left-hand vertical map is evaluation at $i=i$ and hence the left triangle commutes. The righthand vertical map is a weak equivalence because $r_{i} \rightarrow F_{i, a}$ is a trivial cofibration in the injective model structure, while the bottom map is a weak equivalence because it is evaluation at $i$ of the levelwise weak equivalence $\mathcal{B} H \rightarrow R \mathcal{B} H$. It follows that the derived unit $\iota \eta$ is a weak equivalence. 
Example 4.5. Let $I=G$ be a group and $F(*)=H$ be another group. Then $\mathcal{B} F=$ $G \rtimes H$ and Proposition 4.4 gives a Quillen equivalence between $(G \rtimes H)-s \mathcal{S} e t$ and $(G-s \mathcal{S} e t) / B H$.

Example 4.6. Let $I=\Delta_{+}$be the subcategory of $\Delta$ consisting of order-preserving monomorphisms. There is a natural functor $F: \Delta_{+} \rightarrow$ Cat that sends $[n]$ to the $n$-th symmetric group $\Sigma_{n}$. BF is then a skeleton of the category of monomorphisms between finite sets (and hence a subcategory $\Gamma_{+}$of Segal's $\Gamma$-category).

In this case, Proposition 4.4 says that $\Gamma_{+}$-spaces is Quillen equivalent to semicosimplicial spaces over the semicosimplicial space $[n] \rightarrow B \Sigma_{n}$.

Example 4.7. We can regard a simplicial set as a diagram of discrete categories

$$
X: \Delta^{o p} \rightarrow \text { Cat. }
$$

The Grothendieck construction on this functor is the category of simplices of $X$ which we denote $c X$. Proposition 4.4 gives us a Quillen equivalence

$$
s \operatorname{Set}^{c X} \stackrel{\mathcal{B}}{\longrightarrow} s \operatorname{Set}^{\Delta^{o p}} / X .
$$

Remark 4.8. Fixing $F: I^{o p} \rightarrow$ Cat arguments along the lines of the proof of Proposition 4.4 give the following generalizations of Propositions 2.11 and 2.10, and Corollary 2.12:

(i) If $K \in\left(s \operatorname{Set}^{I^{o p}} / N F\right)_{i n j}$ is fibrant, then the counit $B \mathfrak{G} K \rightarrow K$ is a weak equivalence.

(ii) If each map $(i, a) \stackrel{(\mathrm{id}, \alpha)}{\longrightarrow}(i, b)$ is sent by $H \in s \mathcal{S} e t^{B F^{o p}}$ to a weak equivalence, then the derived unit of the adjunction $(\mathcal{B}, \mathfrak{G})$ is a weak equivalence.

(iii) $(\mathcal{B}, \mathfrak{G})$ induces a Quillen equivalence from the localization of $s \mathcal{S e t}{ }^{B F^{o p}}$ with respect to the maps between representables

$$
\left\{r_{i, a} \rightarrow r_{i, b} \mid i \in I, a \rightarrow b \in F(i)\right\}
$$

to $\left(s \operatorname{Set}^{I^{o p}} / N F\right)_{i n j}$.

\section{The adjoint pair $(\mathcal{P}, \mathcal{B})$}

In this section we discuss the left adjoint to $\mathcal{B}$ and apply it to give a proof of a theorem of Dwyer and Kan describing diagrams indexed by an EI-category.

Definition 5.1. Let $\mathcal{C}=$ Cat, sSet, or Grpd and fix $F: I^{o p} \rightarrow$ Cat. We define

$$
\mathcal{P}: \mathrm{e}^{I^{o p}} / F \rightarrow \mathrm{e}^{B F^{o p}}
$$

by the formula

$$
\mathcal{P} G(i, a)=(a / F(i)) \times_{F(i)} G(i) .
$$

We will now prove that the functor $\mathcal{P}$ is a left adjoint for $\mathcal{B}$ when $\mathcal{C}=\mathcal{C}$ at. For $\mathcal{C}=s \mathcal{S}$ et or $\mathcal{G} r p d$ this is the case only when $F$ is a diagram of groupoids. 


\section{Theorem 5.2.}

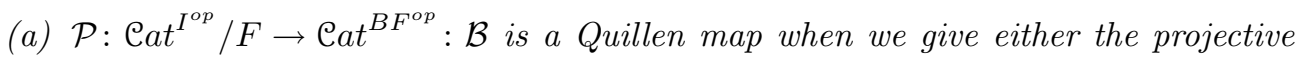
or the injective model structure to both categories.

(b) If $F$ is a diagram of groupoids then, for $\mathrm{C}=s \mathcal{S}$, , $\mathrm{C}$ at and $\mathrm{Grpd}$,

$$
\mathcal{P}: \mathrm{C}^{I^{o p}} / F \rightarrow \mathcal{C}^{B F^{o p}}: \mathcal{B}
$$

is a Quillen equivalence when we give the projective or the injective model structure to both categories.

Proof. We can define the unit and the counit of the adjunctions using the unit and counit in the base case (cf. the proof of Proposition 3.2). For each $(i, a)$ we have the counits of the $(p, B)$-adjunctions

$$
(\mathcal{P B} G)(i, a)=(a / F(i)) \times_{F(i)} G r_{F(i)} G_{\mid F(i)}=\left(p B G_{\mid F(i)}\right)(a) \mapsto G_{\mid F(i)}(a)=G(i, a) .
$$

It is easy to check from the naturality properties of the coend and the pullback that these are natural with respect to maps $(i, a) \rightarrow(j, b)$ and hence yield a map $\mathcal{P B} G \rightarrow G$. Naturality in $G$ is clear.

On the other hand, for each $i$ we have maps

$$
H(i) \rightarrow\left((F(i) /-) \otimes_{F(i)}(-/ F(i))\right) \times_{F(i)} H(i)=(\mathcal{B P} H)(i)
$$

given by the unit of the adjunction in the base case. Again, naturality of the coend and the fiber product implies that these are natural in $i$ and, since they are clearly natural in $H$, they define a natural transformation

$$
\operatorname{id} \rightarrow \mathcal{B P} \in \mathrm{e}^{I^{o p}} / F
$$

These natural transformations satisfy the necessary identities because the ones in the base case do. This proves that $(\mathcal{P}, \mathcal{B})$ is an adjunction. The statements about the model structures follow immediately from the base case.

\subsection{Diagrams indexed by EI-categories}

An EI-category is a category where all endomorphisms are isomorphisms. We now apply Theorem 5.2 to deduce the theorem of Dwyer and Kan [DK2, Theorem 3.4] characterizing diagrams over EI-categories as certain diagrams of fibrations.

Recall [BK, Chapter IX] that a functor $f: I \rightarrow J$ is called right cofinal if the undercategories $N(j / f)$ are contractible for every $j \in J$. Similarly, a functor $f: I \rightarrow J$ is called left cofinal if the overcategories $N(f / j)$ are contractible for every $j \in J$. The main property of right cofinal functors is that given a diagram $X \in s \operatorname{Set}^{J}$, the natural

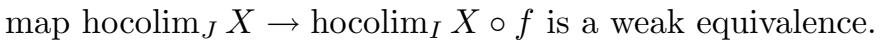

The following proposition is essentially $[\mathbf{D K}, 6.15]$ and we include a proof for the sake of completeness.

Proposition 5.3. Let $f: I \rightarrow J$ be a functor, $L_{f}$ denote the left Kan extension along $f$, and let $c_{i}=\operatorname{Hom}_{I}(i,-) \in$ set $^{I}$. If $f$ satisfies

(a) For each $j \in J$, the nerve $N\left(f^{-1}(j)\right)$ is contractible,

(b) The inclusion $\iota_{j}: f^{-1}(j) \rightarrow j / f$ is left cofinal for each $j \in J$, 
then there is a Quillen equivalence

$$
L_{f}: s \mathcal{S} e t_{p r o j, l o c}^{I} \Leftrightarrow s \mathcal{S e t} t_{p r o j}^{J}: f^{*},
$$

where sSet $t_{\text {proj,loc }}^{I}$ is the localization of $s \operatorname{Set}_{\text {proj }}^{I}$ with respect to the maps of corepresentables

$$
\left\{c_{i} \rightarrow c_{i^{\prime}} \mid f\left(i \rightarrow i^{\prime}\right)=\operatorname{id}_{f(i)}\right\} .
$$

Notice that the fibrant objects in $s \mathcal{S} e t_{\text {proj,loc }}^{I}$ are the diagrams $X$ for which each $X(i)$ is a fibrant simplicial set and which satisfy the following property:

- for all $i \stackrel{\alpha}{\longrightarrow} i^{\prime}$ with $f(\alpha)=i d$ the map $X(i) \rightarrow X\left(i^{\prime}\right)$ is a weak equivalence.

So Proposition 5.3 says that homotopy theory of $J$ diagrams is equivalent the homotopy theory of $I$ diagrams in which send all the maps in the fibers of $f$ to weak equivalences.

Proof. Let $s \mathcal{S} e t_{i n j, l o c}^{I}$ be the localization of the injective model structure $s \mathcal{S} e t_{i n j}^{I}$ with respect to the maps of corepresentables

$$
\left\{c_{i} \rightarrow c_{i^{\prime}} \mid f\left(i \rightarrow i^{\prime}\right)=\operatorname{id}_{f(i)}\right\} .
$$

The fibrant objects in $s \mathcal{S} e t_{i n j, l o c}^{I}$ are the diagrams $X$ which are fibrant in $s \delta e t_{i n j}^{I}$ and which satisfy:

- for all $i \stackrel{\alpha}{\longrightarrow} i^{\prime}$ with $f(\alpha)=i d$ the map $X(i) \rightarrow X\left(i^{\prime}\right)$ is a weak equivalence,

Let $R_{f}$ denote the right Kan extension along $f$. We have a Quillen pair

$$
f^{*}: s \operatorname{Set}_{i n j}^{J} \Leftrightarrow s \operatorname{Set}_{i n j, l o c}^{I}: R_{f} .
$$

If $X$ is fibrant $\operatorname{holim}_{j / f} X \stackrel{\sim}{\longrightarrow} \operatorname{holim}_{f^{-1}(j)} X \stackrel{\sim}{\longrightarrow} X(i)$ for any $i \in f^{-1}(j)$. Since the right-derived functor of $R_{f}$ can be computed by taking the homotopy inverse limit along $j / f$ one sees that both the derived unit and counit of this Quillen pair are weak equivalences and so it is a Quillen equivalence.

The adjunction

$$
L_{f}: s \mathcal{S} e t_{p r o j, l o c}^{I} \Leftrightarrow s \mathcal{S} e t_{\text {proj }}^{J}: f^{*}
$$

is a Quillen pair since $f^{*}$ sends (trivial) fibrations in $s \mathcal{S} e t_{p r o j}^{J}$ to (trivial) fibrations in $s \mathcal{S} t_{\text {proj,loc }}^{I}$.

Notice that we also have a Quillen equivalence given by the identity adjunction

$$
s \mathcal{S} e t_{p r o j, l o c}^{I} \Leftrightarrow s \mathcal{S} e t_{i n j, l o c}^{I}
$$

and so the identity is an isomorphism $H o\left(s S e t_{p r o j, l o c}^{I}\right) \cong H o\left(s \delta e t_{i n j, l o c}^{I}\right)$. Since $f^{*}$ is its own left- and right-derived functor and $\left(f^{*}, R_{f}\right)$ is a Quillen equivalence, it follows that $f^{*}$ also induces an equivalence

$$
H o\left(s \operatorname{Set}_{\text {proj }}^{J}\right) \stackrel{\cong}{\longrightarrow} H o\left(s \operatorname{Set}_{i n j}^{J}\right) \stackrel{f^{*}}{\longrightarrow} H o\left(s \operatorname{Set}_{i n j, l o c}^{I}\right) \stackrel{\cong}{\longrightarrow} H o\left(s \mathcal{S} e t_{\text {proj,loc }}^{I}\right)
$$

and so

$$
L_{f}: s S e t_{p r o j, l o c}^{I} \Leftrightarrow s \operatorname{Set}_{p r o j}^{J}: f^{*}
$$

is also a Quillen equivalence. 
Remark 5.4. The previous lemma gives another example of a homotopy surjection in the sense of Dugger $[\mathbf{D g}]$, as in Remark 2.13.

If $\mathcal{E}$ is an EI-category, let $P$ denote the poset of isomorphism classes in $\mathcal{E}$. Let sd ${ }^{+} P$ the opposite of the poset of non-degenerate simplices of $P$. The objects of $\mathrm{sd}^{+} P$ are the inclusions

$$
\sigma:[k] \rightarrow P,
$$

where $[k]=\{0<1<\cdots<k\}$, and the morphisms $\sigma \rightarrow \sigma^{\prime}$ are commutative triangles

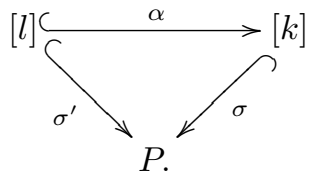

Let

$$
S: \mathrm{sd}^{+} P \rightarrow \operatorname{Grpd}
$$

be the functor assigning to $\sigma \in \mathrm{sd}^{+} P$, the groupoid of sections

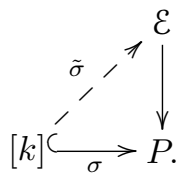

Let $\mathcal{E}^{\prime}$ be the coend $S \otimes_{s d^{+} P}\left(-/ s d^{+} P\right)$. Objects of $\mathcal{E}^{\prime}$ are lifts $\tilde{\sigma}$ (as above) and morphisms $\tilde{\sigma} \rightarrow \tilde{\sigma}^{\prime}$ are pairs $(\alpha, \phi)$ where $\sigma \stackrel{\alpha}{\longrightarrow} \sigma^{\prime}$ and $\phi$ is a natural isomorphism $\tilde{\sigma} \circ \alpha \rightarrow \tilde{\sigma}^{\prime}$.

There is a functor $\pi: \mathcal{E}^{\prime} \rightarrow \mathcal{E}$ given by evaluation at 0 ,

$$
\begin{aligned}
\tilde{\sigma} & \mapsto \tilde{\sigma}(0) \\
(\alpha, \phi) & \mapsto \phi_{\mid \alpha(0)} \circ \tilde{\sigma}(0 \rightarrow \alpha(0)) .
\end{aligned}
$$

Proposition 5.5. Let $\pi: \mathcal{E}^{\prime} \rightarrow \mathcal{E}$ be as above. Then

$$
L_{\pi}: s \operatorname{Set}_{\text {proj }}^{\mathcal{E}^{\prime}} \Leftrightarrow s \mathcal{S} e t_{\text {proj }}^{\mathcal{E}}: \pi^{*}
$$

induces a Quillen equivalence between the localization of $s \delta e t_{\text {proj }}^{\mathcal{E}}$ with respect to the maps of corepresentables

$$
\left\{c_{\tilde{\sigma}^{\prime}} \rightarrow c_{\tilde{\sigma}} \mid \pi\left(\tilde{\sigma} \rightarrow \tilde{\sigma}^{\prime}\right)=\tilde{\sigma}(0) \stackrel{i d}{\longrightarrow} \tilde{\sigma}^{\prime}(0)\right\}
$$

and $s S_{e t}^{\varepsilon} t_{p r o j}$.

Proof. We will check that $\pi$ satisfies the hypothesis of Proposition 5.3. Given $a \in \mathcal{E}$, $\pi^{-1}(a)$ consists of all chains of maps out of $a$ and so has a final object. Let

$$
b=\left(\left[a_{0} \rightarrow a_{1} \rightarrow \cdots \rightarrow a_{n}\right], a \stackrel{f}{\longrightarrow} a_{0}\right)
$$

be an object of $a / \pi$. If $\phi_{a}$ denotes the inclusion $\pi^{-1}(a) \rightarrow a / \pi$, then $\phi_{a} / b$ has a final object which is 
- $\left(\left[a \rightarrow a_{1} \rightarrow \cdots \rightarrow a_{n}\right], a \stackrel{=}{\longrightarrow} a\right) \stackrel{(\mathrm{id}, f), f}{\longrightarrow}\left(\left[a_{0} \rightarrow a_{1} \rightarrow \cdots \rightarrow a_{n}\right], a \rightarrow a_{0}\right)$ when $f$ is an isomorphism

- $\left(\left[a \stackrel{f}{\longrightarrow} a_{0} \rightarrow a_{1} \rightarrow \cdots \rightarrow a_{n}\right], a \stackrel{=}{\longrightarrow} a\right) \stackrel{\left(d_{0}, \mathrm{id}\right), f}{\longrightarrow}\left(\left[a_{0} \rightarrow a_{1} \rightarrow \cdots \rightarrow a_{n}\right], a \rightarrow a_{0}\right)$ when $f$ not an isomorphism.

It follows that $\phi_{a} / b$ is contractible.

Theorem 5.6. Let $W$ be the set of morphisms

$$
\left\{\mathcal{B} c_{\tilde{\sigma}^{\prime}} \rightarrow \mathcal{B} c_{\tilde{\sigma}} \mid \pi\left(\tilde{\sigma} \rightarrow \tilde{\sigma}^{\prime}\right)=\operatorname{id}_{\tilde{\sigma}(0)}\right\}
$$

in $s \operatorname{Set}^{\mathrm{sd}^{+} P} / N\left(S^{o p}\right)$. Let $s \operatorname{Set}^{\mathrm{sd}^{+} P} / N\left(S^{o p}\right)_{\text {proj,loc }}$ denote the localization of

$$
s \operatorname{Set}^{\mathrm{sd}^{+} P} / N\left(S^{o p}\right)_{p r o j}
$$

with respect to $W$. There is a Quillen equivalence

$$
s S_{e t} t^{\mathrm{Sd}^{+} P} / N\left(S^{o p}\right)_{\text {proj,loc }} \longrightarrow s \operatorname{Set}_{\text {proj }}^{\mathcal{E}} .
$$

Proof. Taking $I=\left(\mathrm{sd}^{+} P\right)^{o p}$ and $F=S^{o p}$, we see that

$$
B F^{o p}=\left(\left(\left(\mathrm{sd}^{+} P\right)^{o p} /-\right) \otimes_{\left(\mathrm{sd}^{+} P\right)^{o p}} S^{o p}\right)^{o p} \cong S \otimes_{s d^{+} P}\left(-/ s d^{+} P\right)=\mathcal{E}^{\prime}
$$

and so applying Theorem 5.2(b) we have a Quillen equivalence

$$
\mathcal{P}: s \mathrm{Set}^{\mathrm{sd}^{+} P} / N\left(S^{o p}\right)_{\text {proj }} \Leftrightarrow s \operatorname{Set}_{\text {proj }}^{\mathcal{E}^{\prime}}: \mathcal{B} .
$$

We may localize this to obtain a Quillen equivalence from the localization of

$$
s \mathcal{S e t}^{\mathrm{sd}^{+} P} / N(S)_{\text {proj }}
$$

with respect to the morphisms

$$
\left\{\mathcal{B} c_{\tilde{\sigma}^{\prime}} \rightarrow \mathcal{B} c_{\tilde{\sigma}}: \pi\left(\tilde{\sigma} \rightarrow \tilde{\sigma}^{\prime}\right)=\operatorname{id}_{\tilde{\sigma}(0)}\right\}
$$

to the localization of $s \mathcal{S} e t_{\text {proj }}^{\mathcal{E}^{\prime}}$ with respect to the maps of corepresentables

$$
\left\{c_{\tilde{\sigma}^{\prime}} \rightarrow c_{\tilde{\sigma}}: \pi\left(\tilde{\sigma} \rightarrow \tilde{\sigma}^{\prime}\right)=\operatorname{id}_{\tilde{\sigma}(0)}\right\}
$$

Composing with the Quillen equivalence of Proposition 5.5 we have the desired Quillen equivalence. under which an $\mathcal{E}$ diagram is sent to the $\mathrm{sd}^{+} P$ diagram

$$
\left.\sigma \mapsto X \circ \pi\right|_{S(\sigma)} \otimes_{S(\sigma)}-/ S(\sigma)=B\left(\left.X \circ \pi\right|_{S(\sigma)^{o p}}\right.
$$

Using the $(\mathcal{B}, \mathfrak{G})$ adjunction it is easy to check that the fibrant objects in this localization of $s \mathcal{S} e t^{\mathrm{sd}^{+} P} / N\left(S^{o p}\right)_{\text {proj }}$ are the diagrams $Y$ of fibrations such that for each

$$
\sigma \stackrel{\alpha}{\longrightarrow} \sigma^{\prime} \in \operatorname{sd}^{+} P, \text { with } \alpha(0)=0
$$

the square

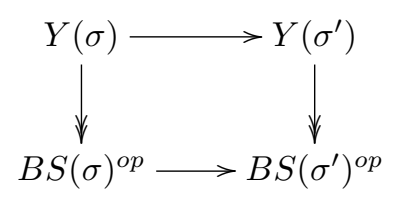

is homotopy cartesian. It follows that the "homotopy theory" of these diagrams is 
equivalent to the homotopy theory of $\mathcal{E}$ diagrams of simplicial sets. So the previous theorem can be interpreted as a model category theoretic version of $[\mathbf{D K 2}$, Theorem 3.4 .

Example 5.7. Let $\mathcal{E}$ be the category with two objects $a_{1}, a_{2}$, and morphisms

$$
\operatorname{End}\left(a_{i}\right)=\operatorname{Aut}\left(a_{i}\right)=G_{i}, \quad \operatorname{Hom}\left(a_{1}, a_{2}\right)=H, \quad \operatorname{Hom}\left(a_{2}, a_{1}\right)=\emptyset .
$$

In this case $P$ is the category with two objects $\overline{a_{1}}, \overline{a_{2}}$ and a single nonidentity morphism $\overline{a_{1}} \rightarrow \overline{a_{2}}$. The subdivision $s d^{+} P$ is the category with three objects and morphisms as follows

$$
\left[a_{1}\right] \leftarrow\left[a_{1} \rightarrow a_{2}\right] \rightarrow\left[a_{2}\right]
$$

Here $S\left(\left[a_{i}\right]\right)$ is the group $G_{i}$ and $S\left(\left[a_{1} \rightarrow a_{2}\right]\right)$ is the groupoid $H_{h\left(G_{1} \times G_{2}\right)}$ with objects $H$ and morphims $h_{1} \rightarrow h_{2}$ consist of pairs $\left(g_{1}, g_{2}\right), g_{i} \in G_{i}$ with $g_{2} \circ h_{1}=h_{2} \circ g_{1} \in$ $\operatorname{Hom}\left(a_{1}, a_{2}\right)$. By Theorem 5.6 the homotopy theory of diagrams of simplicial sets indexed on $\mathcal{E}$ is equivalent to the homotopy theory of diagrams

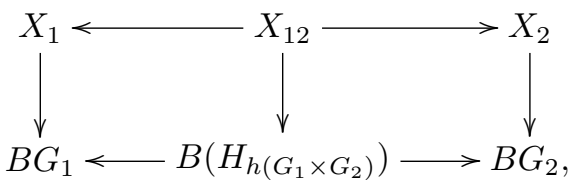

where the first square is homotopy cartesian. Notice that $B\left(H_{h\left(G_{1} \times G_{2}\right)}\right)$ is the homotopy orbit space $H \times{ }_{G_{1} \times G_{2}} E\left(G_{1} \times G_{2}\right)$. The equivalence is determined by sending $Y \in s \mathcal{S e t}^{\mathcal{E}}$ to the diagram

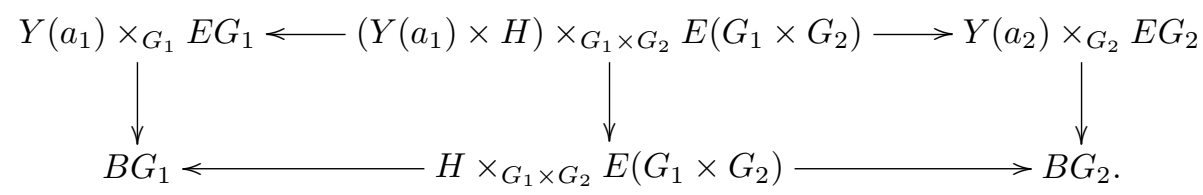

\section{Local model structures}

Let $\mathcal{C}$ be a Grothendieck topology and let $\mathcal{M} \in P(\mathcal{C}, \mathcal{G r p d})$. Recall from [H2] that the fibered site $\mathcal{C} / \mathcal{M}$ is the category $B \mathcal{M}$ with covers the collections of maps whose images are covers in $\mathrm{C}$.

There are local model category structures on $P(\mathcal{C}, \mathcal{G r p d})$ obtained by localizing the injective or projective model structure with respect to the maps

$$
\mathcal{L}_{\mathcal{C}}=\left\{\left|U_{\bullet}\right| \rightarrow X \in P(\mathcal{C}, \mathcal{G r p d})\right\}
$$

where $U_{\bullet}$ is the nerve of a cover $\left\{U_{i} \rightarrow X\right\}$ in $\mathcal{C}$, and $\left|U_{\bullet}\right|$ is the geometric realization of the simplicial diagram of representable functors in $P(\mathcal{C}, \mathcal{G r p d})$.

Similarly, there are local model category structures on $P(\mathcal{C}, s \mathcal{S} e t)$ obtained by localizing the injective or projective model structures with respect to the maps

$$
\mathcal{L}_{\mathcal{C}}=\left\{\left|U_{\bullet}\right| \rightarrow X \in P(\mathcal{C}, s \mathcal{S} e t)\right\}
$$

where $U_{\bullet}$ is a hypercover of $X$ in $\mathcal{C}($ for all this see $[\mathbf{D H I}])$. 
Lemma 6.1. Let $\mathcal{D}=$ sSet or $\mathcal{G} r p d$. The functor $\mathcal{B}: P(\mathcal{C} / \mathcal{M}, \mathcal{D}) \rightarrow P(\mathcal{C}, \mathcal{D}) / \mathcal{M}$ sends fibrations in the local projective model structure to fibrations in the local projective model structure.

Proof. Recall (from [H2, DHI, Proposition 4.2,7.2,7.3]) that $F \rightarrow G \in P(\mathcal{C}, \mathcal{D})$ is a projective local fibration if and only if for all $X \in \mathcal{C}$, the maps

$$
F(X) \rightarrow G(X)
$$

are fibrations and for all (hyper)covers $U \bullet \rightarrow X$ the diagram

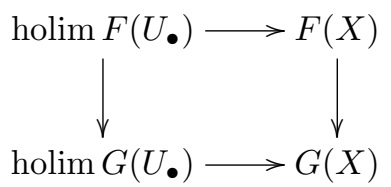

is homotopy cartesian.

We have already observed that $\mathcal{B}$ preserves levelwise fibrations. The second condition follows from the assumption that $F \rightarrow G$ is a projective local fibration, the fact that the squares

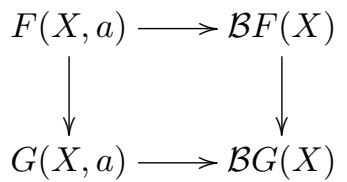

are cartesian and that holim commutes with pullbacks.

The main theorem of this section is the following:

Theorem 6.2. Let $\mathcal{D}=s \mathcal{S}$ et or $\mathcal{G} r p d$, and $\mathcal{M}$ be a presheaf of groupoids on $\mathcal{C}$.

(a) The adjoint pair

$$
\mathcal{B}:(P(\mathcal{C} / \mathcal{M}, \mathcal{D}))_{\text {proj,local }} \longrightarrow(P(\mathcal{C}, \mathcal{D}) / \mathcal{M})_{i_{i n j}, \text { local }}: \mathfrak{G}
$$

is a Quillen equivalence.

(b) The adjoint pair

$$
\mathcal{P}:(P(\mathcal{C}, \mathcal{D}) / \mathcal{M})_{\text {local }} \longrightarrow(P(\mathcal{C} / \mathcal{M}, \mathcal{D}))_{\text {local }}: \mathcal{B}
$$

is a Quillen equivalence when we give both categories either the injective or projective local model structures.

Proof.

(a) Proposition 4.4 tells us that the pairs $(\mathcal{B}, \mathfrak{G})$ are Quillen equivalences for the levelwise model structures.

The topology on $\mathcal{C} / \mathcal{M}$ is generated by the covers of the form

$$
\left\{\left(U_{i}, a \circ u_{i}\right) \stackrel{u_{i}, i d}{\longrightarrow}(X, a)\right\},
$$

where $\left\{U_{i} \stackrel{u_{i}}{\longrightarrow} X\right\}$ is a cover in $\mathcal{C}$ since all other covers in $\mathcal{C} / \mathcal{M}$ are isomorphic to these. 
There is a functorial levelwise weak equivalence

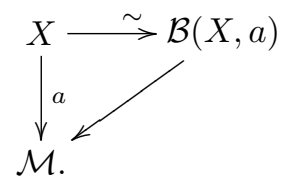

As $\mathcal{B}$ commutes with coproducts, fiber products and geometric realization, it sends the maps in $\mathcal{L}_{\mathcal{C} / \mathcal{M}}$ to weak equivalences. This implies that $(\mathcal{B}, \mathfrak{G})$ descends to a Quillen pair between the local model structures.

We still need to show that the derived unit is a weak equivalence. Given $F \in$ $P(\mathcal{C} / \mathcal{M}, \mathcal{D})$, let $F \rightarrow \widehat{F}$ be a fibrant replacement in the local projective model structure. Let $\mathcal{B}(\widehat{F}) \rightarrow \widehat{\mathcal{B}(\widehat{F})}$ be the injective fibrant replacement. The composite

$$
\mathcal{B} F \rightarrow \mathcal{B} \widehat{F} \rightarrow \widehat{\mathcal{B}(\widehat{F})}
$$

is a local injective fibrant replacement for $\mathcal{B F}$. Hence we can write the derived unit as the composite

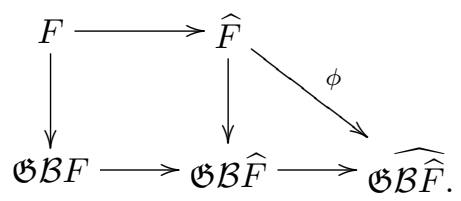

By Lemma $6.1, \mathcal{B} \widehat{F}$ is projective fibrant and therefore the map $\mathcal{B} \widehat{F} \rightarrow \widehat{\mathcal{B F}}$ is a levelwise weak equivalence as it is a local weak equivalence between local projective fibrant objects and hence $\phi$ is the levelwise derived unit applied to $\widehat{F}$. It follows from Proposition 4.4 that $\phi$ is a weak equivalence and hence the whole composite is a local weak equivalence. This completes the proof.

(b) Since $\mathcal{B}$ preserves local weak equivalences and (by Lemma 6.1) projective local fibrations, $(\mathcal{P}, \mathcal{B})$ descends to a Quillen pair between the projective local model structures. An argument similar to the one in $(\mathrm{a})$ shows that $(\mathcal{P}, \mathcal{B})$ is a Quillen equivalence.

$\mathcal{P}$ preserves levelwise cofibrations and so it suffices to show that it sends an injective local trivial cofibration $j$ to a local weak equivalence. Factor $j$ as $p i$ with $p$ a projective local fibration and $i$ a projective local trivial cofibration. Since $(\mathcal{P}, \mathcal{B})$ is a Quillen pair for the projective local model structure, $\mathcal{P}(i)$ is a weak equivalence. Since $p$ is a levelwise weak equivalence, $\mathcal{P}(p)$ is also a weak equivalence.

Note 6.3. This Quillen equivalence induces one between the " $n$-stacks over $\mathcal{M}$ "

$$
P\left(\mathcal{C} / \mathcal{M},\left(S^{n+1}\right)^{-1} s \mathcal{S e t}\right)
$$

and $P\left(\mathcal{C},\left(S^{n+1}\right)^{-1} s \mathcal{S} e t\right) / N \mathcal{M}$.

Corollary 6.4. A weak equivalence $\mathcal{M}^{\prime} \stackrel{\sim}{\longrightarrow} \mathcal{M}$ in $P(\mathcal{C}, \mathcal{G r p d})$ induces a Quillen equivalence between $P\left(\mathrm{C} / \mathcal{M}^{\prime}\right.$, sSet $)$ and $P(\mathcal{C} / \mathcal{M}$, set $)$.

Remark 6.5. If $\mathcal{M}$ is a presheaf of categories on a Grothendieck topology one defines the fibred site in the same way and the pair $(\mathcal{B}, \mathfrak{G})$ will still yield a Quillen pair 
between the corresponding local model structures. This will however not be a Quillen equivalence in general.

\section{References}

[A] M. Artin, Versal deformations and algebraic stacks, Invent. Math. 27 (1974), 165-189.

[B] M. Behrens, A modular description of the $K(2)$-local sphere at the prime 3, Topology 45 (2006), 343-402.

[BK] A. K. Bousfield and D. M. Kan, Homotopy Limits, Completions and Localizations, Lecture Notes in Math. 304, Springer-Verlag, New York (1972).

[DK] W. Dwyer and D. M. Kan, A classification theorem for diagrams of simplicial sets, Topology 23 (1984), 139-155.

[DK2] W. Dwyer and D. M. Kan, Reducing equivariant homotopy theory to the theory of fibrations, Contemp. Math. 37, Amer. Math. Soc., Providence, RI (1985), 35-49.

[DM] P. Deligne and D. Mumford, The irreducibility of the space of curves of given genus, Inst. Hautes Études Sci. Publ. Math. 36 (1969), 75-109.

[Dg] D. Dugger, Universal homotopy theories, Adv. Math. 164 (2001), 144-176.

[DHI] D. Dugger, S. Hollander, and D. C. Isaksen, Hypercovers and simplicial presheaves, Math. Proc. Cambridge Philos. Soc. 136 (2004), 9-51.

[DDK] E. Dror, W. G. Dwyer, and D. M. Kan, Equivariant maps which are self homotopy equivalences, Proc. Amer. Math. Soc. 80 (1980), 670-672.

[Gi] J. Giraud, Cohomologie non abélienne, Springer-Verlag, New York, 1971.

[G] P. Goerss, (Pre-)sheaves of ring spectra over the moduli stack of formal group laws, 101-131, NATO Sci. Ser. II Math. Phys. Chem. 131, Kluwer Acad. Publ., Dordrecht, 2004.

[GHMR] P. Goerss, H.-W. Henn, M. Mahowald, and C. Rezk, A resolution of the K(2)-local sphere at the prime 3, Ann. of Math. 162 (2005), 777-822.

[GJ] P. Goerss and J. F. Jardine, Simplicial Homotopy Theory, Progress in Math. 174, Birkhäuser Verlag, Basel (1999).

[He] A. Heller, Homotopy Theories, Memoirs Amer. Math. Soc. 71, no. 383 (1988).

[Hi] P. S. Hirschhorn, Model Categories and Their Localizations, Mathematical Surveys and Monographs 99, Amer. Math. Soc., Providence, RI, 2003.

[H] S. Hollander, A homotopy theory for stacks, to appear in Israel J. of Math.

[H2] S. Hollander, Descent for quasi-coherent sheaves on stacks, Algebr. Geom. Topol. 7 (2007), 411-437.

[H3] S. Hollander, Stacks and chromatic homotopy theory, in preparation.

[Hp] M. Hopkins, Algebraic topology and modular forms, Proceedings of the International Congress of Mathematicians, Vol. I (Beijing, 2002), 291317, Higher Ed. Press, Beijing, 2002. 
[J] J. F. Jardine, Fibred sites and stack cohomology, Math. Z. 254 (2006), 811-836.

[M] S. MacLane, Categories for the Working Mathematician, Graduate Texts in Math. 5, Springer-Verlag, New York, 1971.

[R] C. Rezk, A model category for categories, preprint available at http:// www. math. uiuc. edu/ rezk/papers.html.

[R2] C. Rezk, Notes on the Hopkins-Miller theorem, in Homotopy Theory via Algebraic Geometry and Group Representations (Evanston, IL, 1997), 313-366, Contemp. Math. 220, Amer. Math. Soc., Providence, RI, 1998.

Sharon Hollander sjh@math.ist.utl.pt

Center for Mathematical Analysis, Geometry and Dynamical Systems, Department of Mathematics, Instituto Superior Técnico, Tech. Univ. Lisbon, Av. Rovisco Pais, 1049-001 Lisboa, Portugal 